Bravo Peña L. C., Torres Olave M. E., Alatorre Cejudo L. C., Castellanos Villegas A. E., Moreno Murrieta R.. L, Granados Olivas A., Uc Campos M., González León M., Wiebe Quintana L. C. (2017): "Áreas probables de degradación-deforestación de la cubierta vegetal en Chihuahua, México. Una exploración mediante regresión logística para el período 1985-2013", GeoFocus (Artículos), $n^{\circ}$ 20, p. 109-137. ISSN: 1578-5157 http://dx.doi.org/10.21138/GF.545

\title{
ÁREAS PROBABLES DE DEGRADACIÓN-DEFORESTACIÓN DE LA CUBIERTA VEGETAL EN CHIHUAHUA, MÉXICO. UNA EXPLORACIÓN MEDIANTE REGRESIÓN LOGÍSTICA PARA EL PERÍODO 1985-2013.
}

\author{
LUIS CARLOS BRAVO PEÑA ${ }^{1}$, MARÍA ELENA TORRES OLAVE ${ }^{1}$, LUIS CARLOS \\ ALATORRE CEJUDO ${ }^{1}$, ALEJANDRO E. CASTELLANOS VILLEGAS ${ }^{2}$, RAMÓN $^{\prime}$ \\ LEOPOLDO MORENO MURRIETA ${ }^{1}$, ALFREDO GRANADOS OLIVAS ${ }^{1}$, MARIO UC \\ CAMPOS $^{1}$, MANUEL GONZÁLEZ LEÓN ${ }^{1}$, LARA CECILIA WIEBE QUINTANA ${ }^{1}$. \\ ${ }^{1}$ Universidad Autónoma de Ciudad Juárez, km 3.5 Carretera Cuauhtémoc-Anáhuac, Cd. Cuauhtémoc CP. \\ 31560, Chihuahua, México. \\ luis.bravo@uacj.mx \\ ${ }^{2}$ Universidad de Sonora. México.Blvd Luis Encinas y Rosales SN, Centro, 83000 Hermosillo, Son., \\ México
}

\section{RESUMEN}

El objetivo de este trabajo fue identificar las áreas con riesgo de degradación-deforestación de la cubierta forestal en el oeste de Chihuahua, en el norte de México, utilizando un modelo de regresión logística. Se generó la regresión entre once capas de información geográfica (biofísica y socioeconómica) y áreas deforestadas de cuatro municipios del estado durante el período 1985-2013. Los resultados indican un buen poder predictivo del modelo generado (Pseudo-R2 de McFadden de 0.24), e identifican la tenencia de la tierra, el grado de marginación y su comportamiento temporal, la aptitud agrológica del suelo y la pendiente del terreno, como las variables con mayor peso en la predicción de zonas susceptibles de degradación y pérdida de bosques. Estos hallazgos facilitan el manejo ambiental de áreas boscosas y la mitigación de causas en zonas que están sufriendo este proceso.

Palabras clave: Deforestación, Chihuahua, Predicción, Regresión Logística.

\section{PROBABLE AREAS OF DEGRADATION-DEFORESTATION OF VEGETATION COVERAGE IN CHIHUAHUA, MEXICO. AN EXPLORATION THROUGH LOGISTIC REGRESSION FOR THE PERIOD 1985-2013.}

\section{ABSTRACT}

The objective of this work was to identify the areas with risk of degradation- deforestation of forest cover in the west of Chihuahua, in the north of Mexico, using a logistic regression model. It was generated the regression between eleven layers of geographic information (biophysical and socioeconomic) and deforested areas of four municipalities of the state during the period 1985-2013. The results indicate a good predictive power of the generated model (McFadden's Pseudo-R2 of 0.24), and identify land tenure, degree of marginalization and its temporal behavior, soil agrological suitability and terrain slope, as the variables with the biggest weight in 
Bravo Peña L. C., Torres Olave M. E., Alatorre Cejudo L. C., Castellanos Villegas A. E., Moreno Murrieta R.. L, Granados Olivas A., Uc Campos M., González León M., Wiebe Quintana L. C. (2017): "Áreas probables de degradación-deforestación de la cubierta vegetal en Chihuahua, México. Una exploración mediante regresión logística para el período 1985-2013", GeoFocus (Artículos), $n^{\circ}$ 20, p. 109-137. ISSN: 1578-5157 http://dx.doi.org/10.21138/GF.545

the prediction of susceptible areas to forest degradation and loss. These findings facilitate the environmental management of forested areas, and mitigation of causes in zones that are suffering this process.

Key words: Deforestation, Chihuahua, Prediction, Logistic Regression.

\section{Introducción.}

En México la deforestación de coberturas en áreas de importancia forestal es un problema muy preocupante. Estimaciones a principios de la década pasada mostraron cifras de hasta $775,800 \mathrm{ha} / \mathrm{año}$ de pérdida de cubierta vegetal forestal (Velázquez et al, 2002), asociado a factores como la ampliación de la frontera agropecuaria (nuevas áreas agrícolas y de pastizal), la intensificación del uso del suelo, los desmontes ilegales y los incendios forestales, que pueden estar relacionados con las actividades agropecuarias, y últimamente con actividades de carácter ilícito, como el narcotráfico. En Chihuahua, una entidad del norte del país con importante cobertura forestal, una estimación con datos de los inventarios forestales durante el periodo 19852008, mostró niveles de pérdida que destacan en el territorio nacional. Tan solo en el bosque de coníferas primario, se perdieron casi $10000 \mathrm{~km}^{2}$ en ese periodo, pues dicha cobertura pasó de $25522 \mathrm{~km}^{2}$ a 15,942 $\mathrm{km}^{2}$ en veintidós años (Pérez et al, 2013).

En Chihuahua este proceso de pérdida de las cubiertas vegetales se relaciona con la ampliación de pastizales y áreas agrícolas en las zonas serranas, pero no está clara la importancia específica que representa cada causa en las distintas zonas del estado. Se desconoce en qué medida los cambios de uso del suelo se favorecen por la cercanía de áreas agrícolas, carreteras, los caminos rurales de terracería, la pendiente del terreno, la aptitud agrológica del suelo, etc.; por lo que es necesario identificar y cuantificar la importancia relativa de estos factores para impulsar medidas que disminuyan la degradación o alteración de cubiertas naturales. Las exploraciones de este tipo deben ser lo suficientemente específicas como para revelar qué pasa en las distintas zonas de la entidad, pues la importancia de los factores causales puede ser diferente dependiendo de las condiciones físicas, económicas, culturales o políticas de cada región de Chihuahua.

Esta preocupación no es nueva, y ha sido motivo de trabajo científico en otras latitudes del mundo desde hace décadas (Mertens et al, 2004). A fines del siglo pasado por ejemplo, Angelsen \& Kaimowitz (1999), mencionaron más de 150 modelos para entender qué factores conducen el proceso de deforestación. Estos autores encontraron que la mayoría de los modelos combinaban en general 115 variables, pero presentaban a menudo resultados contradictorios sobre el mismo proceso. Al final concluyen que esto se asociaba con la complejidad del fenómeno que pretendían modelar. Dicha complejidad convierte a este tipo de exploraciones, uno de los retos científicos más interesantes en geografía (Basse et al, 2014).

En respuesta a esta necesidad, distintos autores han realizado exploraciones para entender los factores causales de fenómenos como la deforestación de bosques y selvas, ampliación de la frontera agrícola, y el cambio de uso del suelo, a partir de establecer las relaciones que estos procesos presentan con variables explicativas que se asumen como variables causales (Pérez- 
Bravo Peña L. C., Torres Olave M. E., Alatorre Cejudo L. C., Castellanos Villegas A. E., Moreno Murrieta R.. L, Granados Olivas A., Uc Campos M., González León M., Wiebe Quintana L. C. (2017): "Áreas probables de degradación-deforestación de la cubierta vegetal en Chihuahua, México. Una exploración mediante regresión logística para el período 1985-2013”, GeoFocus (Artículos), $n^{\circ}$ 20, p. 109-137. ISSN: 1578-5157 http://dx.doi.org/10.21138/GF.545

Vega, Mas, \& Ligmann-Zielinska, 2012; Liénard, Gravel, \& Strigul, 2015). Dentro de estas exploraciones, los modelos sustentados en la técnica de regresión logística (RLO) se utilizan ampliamente para identificar la contribución de variables explicativas a los cambios en los usos del suelo (Pir, 2015; Kumar, Nandy, Agarwal, \& Kushwaha, 2014; Arekhi, 2013), y en el caso específico de procesos como la deforestación de bosques, han probado su utilidad para predecir la pérdida de cobertura e identificar los factores socio-económicos o biofísicos vinculados con este fenómeno (Roy, 2006; Morales-Barquero, Borrego, Skutsch, Kleinn, \& Healey, 2015).

El método de RLO, permite establecer la importancia relativa de las causas o fuerzas motrices que inducen el deterioro de la cubierta vegetal. Constituye un procedimiento estadístico que puede realizarse en un entorno de Sistemas de Información Geográfica (SIGs), pues estos permiten el manejo de grandes capas de información espacial. Los SIGs en conjunto con los sistemas de sensoría remota facilitan la identificación de patrones espaciales no evidentes a simple vista, mediante el procesamiento simultáneo de capas de información geográfica. Constituyen una valiosa herramienta de apoyo para la toma de decisiones, y han probado su utilidad en gran diversidad de condiciones geográficas para el monitoreo de muchos ambientes distintos (Shalaby \& Tateishi, 2007; Marín, Nahuelhual, Echeverría, \& Grant, 2013). Así, la regresión logística realizada en un entorno de SIG, ha permitido la gestión de medidas encaminadas a la protección de ecosistemas (Fagerholm et al., 2016;. Semeraro et al., 2016), y en particular al manejo de bosques (Zambelli et al., 2012; Eugenio et al., 2016; Lin, Thomson, Hung, \& Lin, 2012).

Como método estadístico RLO permite determinar la relación existente entre una variable dependiente, de tipo nominal, y dicotómica, y un conjunto de variables independientes, cuantitativas o categóricas (Rogerson, 2010). Constituye un procedimiento estadístico que tiene cierto parecido con la regresión lineal múltiple (RLM), pero que a diferencia de esta, presenta una variable de respuesta que no tiene distribución continua en términos probabilísticos. Por el contrario, en RLO la variable dependiente es discreta, o categórica, y toma valores de 0 y 1 , en función de la no ocurrencia del fenómeno (Valor 0) o la ocurrencia del mismo (Valor 1). Adicionalmente, las variables explicativas pueden ser cualitativas o cuantitativas, y la ecuación del modelo corresponde a una función exponencial, de tipo logit. En este caso, al aplicar un modelo de RLO, no se construye un modelo lineal para calcular los valores reales de la variable de interés, sino que se calcula la probabilidad de que la variable de respuesta adopte el valor del evento modelado, en función del valor que presentan las variables independientes en la misma ubicación espacial. En el proceso de modelización, el RLO genera coeficientes individuales para cada variable, que están relacionadas con su peso en el cambio de la variable predicha.

Con base en los elementos anteriores, el objetivo de este trabajo fue identificar las zonas con riesgo de deforestación en el poniente de Chihuahua mediante un modelo de regresión logística, que identifique áreas potenciales para la ocurrencia de este fenómeno y la contribución y el peso estadístico de las variables socioeconómicas y ambientales involucradas en este proceso. Para la modelización de la deforestación se consideraron las tendencias de pérdida de cubierta vegetal de bosques de pino, pino-encino, encino-pino y encino (Pinus chihuahuana, Pinus durangensis, Pinus engelmanii, Quercus rugosa, Querqus chihuahuensis, Querqus sideroxyla; Lebgue, Quintana y Soto, 2015) durante el periodo 1985-2013 en la misma zona de estudio, desarrollándose el trabajo en un entorno de Sistemas de Información Geográfica. 
Bravo Peña L. C., Torres Olave M. E., Alatorre Cejudo L. C., Castellanos Villegas A. E., Moreno Murrieta R.. L, Granados Olivas A., Uc Campos M., González León M., Wiebe Quintana L. C. (2017): "Áreas probables de degradación-deforestación de la cubierta vegetal en Chihuahua, México. Una exploración mediante regresión logística para el período 1985-2013", GeoFocus (Artículos), $n^{\circ}$ 20, p. 109-137. ISSN: 1578-5157 http://dx.doi.org/10.21138/GF.545

\section{Materiales y Métodos.}

\section{1 Área de estudio.}

El área de estudio comprende cuatro municipios de la región centro-poniente de Chihuahua (Figura 1), en el norte de México; en una región donde se han apreciado cambios importantes de uso del suelo desde la década de los noventas del siglo pasado (Aguilar, 2013). Estos cuatro municipios (Riva Palacio, Cuauhtémoc, Guerrero y Ocampo) abarcan una superficie de $13439.8 \mathrm{~km}^{2}$, que representa $5.4 \%$ de la extensión territorial del entidad $\left(247087 \mathrm{~km}^{2}\right)$. Esta superficie parece poco en el contexto estatal, pero es superior a la superficie de otros estados en México, como Aguascalientes, Colima, Tlaxcala y Querétaro por separado.

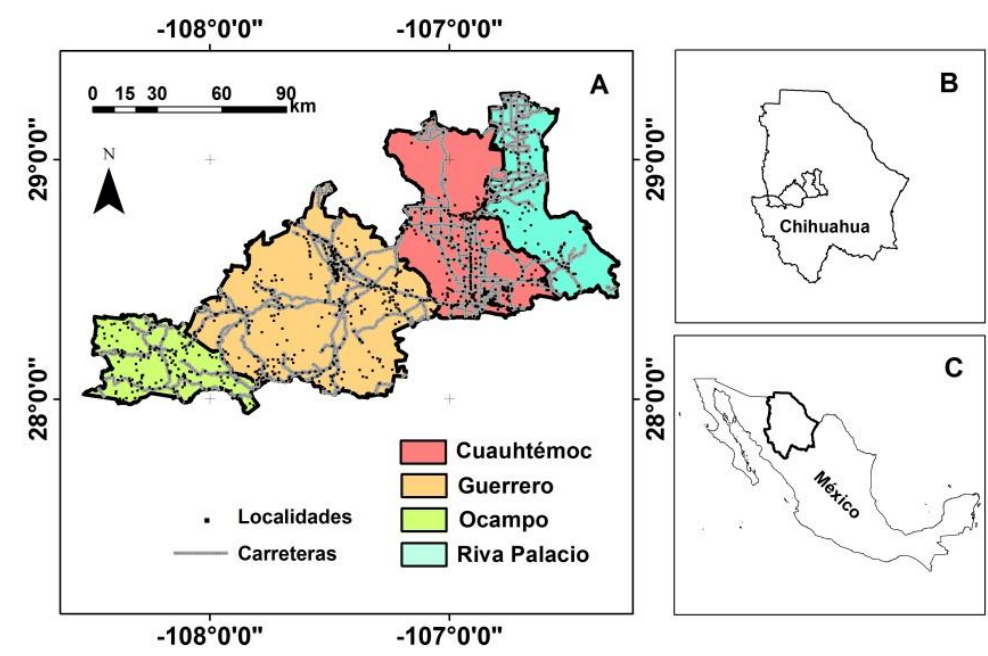

\section{Figura 1. Macro-localización del área de estudio. A) Municipios bajo estudio, B) Ubicación en Chihuahua, C) Ubicación de Chihuahua en México.}

(Fuente: elaboración propia)

El área de estudio se localiza en la vertiente oriental de la Sierra Madre Occidental, formación montañosa que atraviesa Chihuahua, y el resto del país, de noroeste a sureste. Derivada de esta ubicación, el área presenta un rango altitudinal que va de los 1600 metros sobre el nivel del mar (msnm) hacia el este, donde predominan las pendientes más planas, y hasta 2700 msnm en el oeste, donde predomina el relieve más accidentado. Asociado con esta altitud, en el área se observan climas que van desde semi-seco templado en las regiones de menor altitud, hasta subhúmedo semifrío donde las altitudes son mayores (García 1973). Esta combinación de altitud y clima, genera un mosaico de condiciones ambientales que favorecen la presencia de matorrales xerófilos, pastizales, y parches de bosque de encino pino al este, e importantes masas forestales de encino-pino y pino al oeste (Rzedowski, 1978).

En cuanto a condiciones socioeconómicas, en la zona de estudio se localizan 1002 localidades. Según el XII Censo General de Población y Vivienda (INEGI, 2012), estas localidades sumaron 207605 habitantes, con poblaciones que van desde 1 hasta 114,007 personas. En general se trata de poblaciones muy heterogéneas en tamaño, equipamiento e indicadores de desarrollo humano, pero de manera general configuran un patrón espacial que presenta mayor densidad de localidades, y bajos niveles de pobreza al este del área de estudio, y 
Bravo Peña L. C., Torres Olave M. E., Alatorre Cejudo L. C., Castellanos Villegas A. E., Moreno Murrieta R.. L, Granados Olivas A., Uc Campos M., González León M., Wiebe Quintana L. C. (2017): "Áreas probables de degradación-deforestación de la cubierta vegetal en Chihuahua, México. Una exploración mediante regresión logística para el período 1985-2013", GeoFocus (Artículos), $n^{\circ}$ 20, p. 109-137. ISSN: 1578-5157 http://dx.doi.org/10.21138/GF.545

menor densidad de localidades, y altos niveles de pobreza al oeste de la misma, en las zonas serranas. En estas últimas, las condiciones orográficas han propiciado históricamente el aislamiento de las poblaciones, poca diversificación productiva, y carencias importantes en materia de equipamiento urbano, disponibilidad de infraestructura educativa y de salud.

En la zona son muy importantes la ganadería de bovinos y la actividad agrícola. Esta última se desarrolla bajo dos modalidades: como agricultura de temporal en las zonas donde la pendiente y lo accidentado del terreno no permite la construcción o instalación de infraestructura para riego, y bajo la modalidad de riego con agua subterránea, donde la pendiente es lo suficientemente plana, y los predios lo suficientemente grandes, como para pagar las inversiones realizadas en el equipamiento de pozos profundos. La agricultura de temporal usualmente se desarrolla en las vegas de ríos y arroyos, donde se ha acumulado suficiente cantidad de aluvión y materia orgánica, como para trabajar la tierra con pocos insumos químicos después de la temporada de lluvias (Junio, Julio y Agosto). Los predios dedicados a la agricultura de temporal, usualmente se dejaban descansar por lapsos que iban de tres a siete años cada tres ciclos de cultivo, con el fin de que recuperaran su productividad (Hillerkus, 1992). Lo anterior implicaba abrir nuevos terrenos agrícolas en las áreas cubiertas por vegetación natural, que se dejaban en descanso al cabo de tres o cuatro ciclos agrícolas, tiempo durante el cual se utilizaban como potreros ganaderos. Esta forma de trabajar la tierra permitía cierta recuperación de la vegetación original en años o periodos lluviosos, en particular si soportaban poca carga ganadera, o se les abandonaba por muchos años.

Los periodos de descanso a la tierra bajo la modalidad anterior son cada vez menos frecuentes, básicamente por el aumento de la población, que demanda más producción agrícola y más tierra; y por la presión ejercida por cultivos ilícitos (marihuana y amapola) y la tala ilegal, que tienen una presencia creciente hacia el oeste y el sur de la zona de estudio. En este último caso, ocurre que las bandas del narcotráfico también incursionan en el tráfico ilegal de madera, fomentando la tala del bosque, y el incendio de las áreas deforestadas (Publicación Vida Nueva, 2015; Periódico Zócalo, 07/05/2016), para que una vez quemadas se borre la evidencia del desmonte -al quemarse los tocones no hay elementos para sustentar tala ilegal-. La extracción de madera o leña ilegal reporta jugosas ganancias, pero es una práctica que requiere construir una cadena de complicidades, por lo que no se observa el cambio de cubiertas de un día para otro. En lugar de eso, la cubierta vegetal se va degradando gradualmente, conforme se extrae biomasa en forma de leña, o se cortan los árboles de gran talla y los predios deforestados se incendian.

Debido a las presiones anteriores, en la zona pueden observarse predios con cubierta forestal en diferentes condiciones de calidad ecológica o degradación. Al lado de áreas relativamente conservadas, con poca evidencia de disturbio humano, es posible encontrar predios donde el bosque tiene una calidad ecológica menor, pues predominan los arboles de poca talla, el bosque está muy disperso, predominan matorrales y vegetación secundaria, o de plano no hay vegetación porque el predio se ha degradado hasta un punto irreversible. La coexistencia y vecindad espacial de estas tres condiciones ecológicas de calidad de la vegetación original, y la dinámica de los usos del suelo predominantes en la zona, revelan que el proceso de cambio de coberturas forestales a no forestales, puede involucrar el cambio abrupto de terreno forestal a no forestal, pero frecuentemente involucra una degradación paulatina de las áreas forestales, siguiendo la trayectoria a) de áreas boscosas a áreas degradadas donde predomina el matorral y vegetación secundaria, y posteriormente en terrenos con uso agrícola o de pastizal; o bien la trayectoria b) de áreas boscosas a terrenos agrícolas, que se abandonan por muchos años y se 
Bravo Peña L. C., Torres Olave M. E., Alatorre Cejudo L. C., Castellanos Villegas A. E., Moreno Murrieta R.. L, Granados Olivas A., Uc Campos M., González León M., Wiebe Quintana L. C. (2017): "Áreas probables de degradación-deforestación de la cubierta vegetal en Chihuahua, México. Una exploración mediante regresión logística para el período 1985-2013", GeoFocus (Artículos), $n^{\circ}$ 20, p. 109-137. ISSN: 1578-5157 http://dx.doi.org/10.21138/GF.545

convierten en áreas con vegetación secundaria, o eventualmente, en casos raros, en bosque regenerado.

\subsection{Identificación de áreas degradadas-deforestadas.}

En atención a las condiciones prevalecientes en la zona, en este trabajo se decidió considerar a la degradación-deforestación de cubiertas forestales como dos partes de un mismo proceso. En consecuencia, en la generación de los insumos para la modelación mediante RLO se consideraron tanto las áreas deforestadas en un periodo determinado, como los áreas que experimentaron degradación evidente de la cubierta vegetal en el mismo periodo, aunque no hayan experimentado un cambio abrupto de condición forestal a no forestal.

Para la identificación de los predios en cualquiera de las dos condiciones anteriores, se utilizaron las cartografías de uso del suelo y vegetación generadas por la Comisión de Estudios de Territorio Nacional (CETENAL) en 1985, publicada en formato vectorial por INEGI (2001) Uso del suelo y Vegetación Serie I-, y la cartografía digital de Uso del suelo y vegetación serie $\mathrm{V}$, que es la más reciente cartografía a nivel nacional de cubierta vegetal y de uso del suelo, publicada por INEGI (2013b). Ambas cartografías se desarrollaron bajo la misma base teórica y metodológica, mediante reconocimientos de campo y fotointerpretación en imágenes de satélite (INEGI, 2015), por lo que en este trabajo se realizó una homogenización de las clases de usos de suelo y cubierta vegetal para facilitar la comparación entre ambas.

Previo a la homogenización, se verificó la correspondencia espacial de clases y coberturas de uso del suelo en ambas cartografías. Aunque hay consistencia metodológica en los distintos inventarios (INEGI, 2015), la escala en la que fueron generados (1:250 000), y la disponibilidad de insumos satelitales de mayor calidad en fechas recientes, puede facilitar confusiones en la comparación de un año a otro, especialmente cuando se realizan comparaciones con los inventarios más antiguos. Se encontró que la cobertura de asentamientos humanos en la cartografía de 1985, omitía localidades que si aparecen en la cartografía de 2013, básicamente por el tamaño de los asentamientos, que no eran visibles o fácilmente cartografiables en las imágenes satelitales disponibles en la época (Landsat MSS). Debido a eso, para incrementar la precisión en las comparaciones, se incorporaron a la cartografía más antigua los polígonos de los asentamientos omitidos. Este proceso implicó la fotointerpretación de ortofotos aéreas de la época (resolución por pixel de $2 \mathrm{~m}$ ), tomando como base los polígonos de los asentamientos si incluidos en el mapa más reciente. Dichos polígonos fueron redelimitados en plataforma de SIG, con base en el tamaño y la forma de los poblados en la década de los 80's, para ser añadidos posteriormente -mediante algebra de mapas- al inventario de uso del suelo de 1985. Además de esta operación, se verificó la base de atributos tabulares correspondientes a cada mapa, con el propósito de identificar y eliminar inconsistencias y omisiones en el etiquetado de los polígonos.

Una vez concluido el proceso anterior, ambas cartografías fueron reclasificadas en 2 clases: Natural y Antrópica. En la clase "Natural", se incluyeron las categorías bosque de coníferas, bosque de coníferas y latifoliadas, bosque de latifoliadas, matorral xerófilo, otros tipos de vegetación, pastizales naturales, selva caducifolia y selva sub- caducifolia; que incluyen básicamente vegetación primaria en buen estado de conservación ecológica y poca presencia de elementos vegetales correspondientes a vegetación secundaria (arbustiva, herbácea o arbórea) producto de disturbios humanos -se trata de clases con vegetación primaria dominante, con presencia marginal de vegetación secundaria-. Por el contrario, en la clase "Antrópicas" se 
Bravo Peña L. C., Torres Olave M. E., Alatorre Cejudo L. C., Castellanos Villegas A. E., Moreno Murrieta R.. L, Granados Olivas A., Uc Campos M., González León M., Wiebe Quintana L. C. (2017): "Áreas probables de degradación-deforestación de la cubierta vegetal en Chihuahua, México. Una exploración mediante regresión logística para el período 1985-2013", GeoFocus (Artículos), $n^{\circ}$ 20, p. 109-137. ISSN: 1578-5157 http://dx.doi.org/10.21138/GF.545

incluyeron las categorías: agricultura de temporal, pastizales inducidos y cultivados, agricultura de riego y humedad, asentamientos humanos, y áreas donde la vegetación primaria se ha degradado significativamente, substituyéndose de forma irreversible por vegetación secundaria debido a la presión antrópica constante (categoría: sólo vegetación secundaria), o bien áreas donde la vegetación primaria se ha deforestado, sin que se observen procesos de recuperación ecológica y repoblación vegetal (categoría: sin vegetación aparente). Esta reclasificación tomó como base los atributos tabulares de cada mapa, y se hizo con el propósito de que los cruces de ambos insumos y las estadísticas espaciales derivadas fueran equivalentes y comparables.

Posterior a la reclasificación de las cartografías, se construyó una matriz de tabulación cruzada (CROSSTAB), en el programa IDRISI selva, con base en la metodología propuesta por Pontius, Shusas, \& McEachern (2004). La tabulación cruzada consiste en una tabla o matriz de doble entrada, donde se comparan las coberturas y las clases de interés de un año a otro, para identificar los cambios en el periodo evaluado. En dicha matriz, al final de las columnas se observa el área de la clase a en el tiempo uno y al final de los renglones el área de la misma clase a en el tiempo dos, mientras que en la diagonal de la matriz se identifican las superficies constantes de un tiempo a otro. A los costados de la diagonal se encuentran las superficies que cambiaron: áreas sumadas a la clase si están en los renglones, o restadas a la misma si están en las columnas. Esta misma lógica aplica para las clases restantes (clases b,c,d hasta n).

\subsection{Identificación y caracterización de variables explicativas.}

El proceso de degradación-deforestación se vincula con un conjunto de variables explicativas. En la bibliografía se reporta usualmente a variables socioeconómicas como tenencia de la tierra, existencia de subsidios, distancia a caminos, pobreza, áreas agrícolas actuales etc.; mientras que en las variables físicas destaca la utilización de variables como pendiente, distancia a cuerpos de agua y escurrimientos, etc. (Serra, Pons, \& Saurí, 2008; Pineda Jaimes, Bosque Sendra, Gómez Delgado, \& Plata Rocha, 2009; Morales-Barquero et al., 2015); Partiendo de estos antecedentes, y del conocimiento que se tiene de la zona, en este trabajo se utilizaron once variables explicativas del proceso, vinculadas a las dinámicas históricas y actuales de ocupación del territorio en los municipios con cubierta forestal. Estas variables, los procesos a las que se sometieron en el SIG y sus fuentes se indican en la tabla 1.

Tabla 1. Variables y procesos desarrollados en el trabajo.

\begin{tabular}{|c|c|c|c|}
\hline Variable & $\begin{array}{c}\text { Acró- } \\
\text { nimo }\end{array}$ & Fuente & Proceso desarrollado en este trabajo. \\
\hline $\begin{array}{c}\text { Aptitud } \\
\text { agrológica del } \\
\text { suelo }\end{array}$ & CEd & $\begin{array}{c}\text { Carta Edafológica de la } \\
\text { cartografía de INEGI } \\
(2007) .\end{array}$ & $\begin{array}{c}\text { Se obtuvo al reclasificar la cartografía de suelos } \\
\text { de INEGI (INEGI, 2007a) en grupos de aptitud } \\
\text { agrológica, con base en sus atributos texturales, } \\
\text { morfométricos, físico-químicos, y de respuesta } \\
\text { hidrológica. } \\
\text { Estos grupos fueron: }\end{array}$ \\
& $\begin{array}{c}\text { Feozems (4: Alta aptitud), Regosol, Planosoles, } \\
\text { Vertisoles Xerosoles (3: Media aptitud), } \\
\text { Luvisoles y Cambisoles (2: Baja aptitud); } \\
\text { Litosol (1: Muy baja aptitud) }\end{array}$ \\
\hline Pendiente & Pe & Modelo Digital de & El MDE se clasificó en niveles de pendiente en \\
\hline
\end{tabular}


Bravo Peña L. C., Torres Olave M. E., Alatorre Cejudo L. C., Castellanos Villegas A. E., Moreno Murrieta R.. L, Granados Olivas A., Uc Campos M., González León M., Wiebe Quintana L. C. (2017): "Áreas probables de degradación-deforestación de la cubierta vegetal en Chihuahua, México. Una exploración mediante regresión logística para el período 1985-2013", GeoFocus (Artículos), $n^{\circ}$ 20, p. 109-137. ISSN: 1578-5157 http://dx.doi.org/10.21138/GF.545

\begin{tabular}{|c|c|c|c|}
\hline & & $\begin{array}{l}\text { Terreno (Continuo de } \\
\text { Elevaciones Mexicano) } \\
\quad \text { (INEGI, 2013a) }\end{array}$ & $\begin{array}{l}\text { grados. } \\
\text { El producto resultante se clasificó en cinco } \\
\text { clases de aptitud para las actividades } \\
\text { agropecuarias: } 5 \text {, Pendiente menor a } 3 \text { grados, } 4 \\
\text {, pendiente entre } 3 \text { y } 5 \text { grados; } 3 \text {, pendiente entre } \\
5 \text { y } 10 \text { grados; } 2 \text {, pendiente entre } 10 \text { y } 30 \\
\text { grados; } 1 \text {, pendiente mayor a } 30 \text { grados. }\end{array}$ \\
\hline $\begin{array}{l}\text { Distancia a } \\
\text { arroyos y } \\
\text { escurrimientos } \\
\text { superficiales. }\end{array}$ & DEsc & $\begin{array}{l}\text { Mapa de arroyos y } \\
\text { escurrimientos } \\
\text { superficiales. } \\
\text { Fotointerpretación en } \\
\text { este trabajo. }\end{array}$ & $\begin{array}{l}\text { Se generó un mapa de distancia mediante } \\
\text { operadores de contexto espacial. }\end{array}$ \\
\hline $\begin{array}{l}\text { Distancia a } \\
\text { polígonos } \\
\text { agrícolas en } \\
1985\end{array}$ & DPA & $\begin{array}{l}\text { Inventario de uso del } \\
\text { suelo y vegetación } \\
\text { correspondiente a } 1985 . \\
\text { (INEGI, 2001) }\end{array}$ & $\begin{array}{c}\text { Se generó un mapa de distancia a predios } \\
\text { agrícolas que aparecen en la cartografía de } \\
\text { 1985, mediante operadores de contexto espacial. }\end{array}$ \\
\hline $\begin{array}{l}\text { Distancia a } \\
\text { carreteras }\end{array}$ & DCA & $\begin{array}{c}\text { Mapa digital de } \\
\text { Carreteras y publicado } \\
\text { en la cartografía de } \\
\text { INEGI, actualizado } \\
\text { mediante } \\
\text { fotointepretación } \\
\end{array}$ & $\begin{array}{l}\text { Se generó un mapa de distancia mediante } \\
\text { operadores de contexto espacial. }\end{array}$ \\
\hline $\begin{array}{l}\text { Distancia } \\
\text { caminos de } \\
\text { terraceria }\end{array}$ & Dbr & $\begin{array}{l}\text { Mapa de caminos de } \\
\text { terracería generado en } \\
\text { este trabajo } \\
\text { (fotointerpretación) }\end{array}$ & $\begin{array}{l}\text { Se generó un mapa de distancia mediante } \\
\text { operadores de contexto espacial. }\end{array}$ \\
\hline $\begin{array}{l}\text { Distancia a } \\
\text { localidades }\end{array}$ & DLoc & $\begin{array}{l}\text { Mapa de Localidades, } \\
\text { obtenido del XII Censo } \\
\text { General de Población y } \\
\text { Vivienda (INEGI, } \\
\text { 2010). }\end{array}$ & $\begin{array}{l}\text { Se generó un mapa de distancia mediante } \\
\text { operadores de contexto espacial. }\end{array}$ \\
\hline $\begin{array}{l}\text { Distancia a } \\
\text { pozos. }\end{array}$ & $\begin{array}{l}\text { DRepd } \\
\mathrm{a}\end{array}$ & $\begin{array}{l}\text { Mapa ubicación de } \\
\text { pozos de extracción de } \\
\text { agua subterránea } \\
\text { (Registro Público de los } \\
\text { Derechos de Agua } \\
\text { 2015). }\end{array}$ & $\begin{array}{l}\text { Se generó un mapa de distancia mediante } \\
\text { operadores de contexto espacial. }\end{array}$ \\
\hline $\begin{array}{l}\text { Tenencia de la } \\
\text { Tierra }\end{array}$ & $\mathrm{Te}$ & $\begin{array}{l}\text { Mapa de tenencia de la } \\
\text { tierra (Registro Agrario } \\
\text { Nacional, 2012). }\end{array}$ & $\begin{array}{l}\text { Se reclasificó la tenencia de la tierra en } \\
\text { propiedad federal (1), ejidal (2) y privada (3). }\end{array}$ \\
\hline $\begin{array}{l}\text { Marginación } \\
1995\end{array}$ & Marg & $\begin{array}{l}\text { Mapa de Índice de } \\
\text { Marginación,1995 } \\
\text { (CONABIO,2001) } \\
\end{array}$ & $\begin{array}{c}\text { Se generó un mapa de superficies de } \\
\text { marginación mediante el método de polígonos } \\
\text { de Thiessen. }\end{array}$ \\
\hline $\begin{array}{l}\text { Tendencias de } \\
\text { la marginación } \\
(1995-2010)\end{array}$ & CMarg & $\begin{array}{l}\text { Se generó un mapa de } \\
\text { índice marginación con } \\
\text { los estadísticos a nivel } \\
\text { localidad } \\
\text { correspondientes al año } \\
\text { 2010. (CONABIO, } \\
\text { 2012). }\end{array}$ & $\begin{array}{l}\text { Este mapa resulta de restar mediante algebra de } \\
\text { mapas, el mapa de marginación 2010, al mapa } \\
\text { de marginación correspondiente a } 1995 .\end{array}$ \\
\hline
\end{tabular}

(Fuente: elaboración propia) 
Bravo Peña L. C., Torres Olave M. E., Alatorre Cejudo L. C., Castellanos Villegas A. E., Moreno Murrieta R.. L, Granados Olivas A., Uc Campos M., González León M., Wiebe Quintana L. C. (2017): "Áreas probables de degradación-deforestación de la cubierta vegetal en Chihuahua, México. Una exploración mediante regresión logística para el período 1985-2013”, GeoFocus (Artículos), $n^{\circ}$ 20, p. 109-137. ISSN: 1578-5157 http://dx.doi.org/10.21138/GF.545

Las variables anteriores se generaron en el trabajo mediante fotointerpretación en imágenes de alta resolución, o bien se obtuvieron de cartografía publicada. Por lo que se refiere a variables de tipo biofísico como la aptitud agrológica del suelo y la pendiente del terreno, estas fueron subdivididas en rangos. La aptitud agrológica por ejemplo, se subdividió en cuatro categorías, considerando los atributos texturales y de composición química de los suelos presentes en el área de estudio (Tabla 2). La pendiente, por su parte, obtenida del Modelo Digital de Elevación del Terreno provisto en continuo de elevaciones mexicano 3.0 (INEGI, 2012), fue subdividida en cinco categorías (Tabla 3) según las referencias de Priego et al (2008), quien divide la pendiente en función de su aptitud para el desarrollo de las actividades agropecuarias.

Tabla 2. Superficie de las clases de aptitud agrológica del suelo.

\begin{tabular}{|c|c|r|r|}
\hline $\begin{array}{c}\text { Categoría } \\
\text { de Aptitud }\end{array}$ & Tipo de Suelo & \multicolumn{1}{c|}{ Superficie ha } & \multicolumn{1}{|c|}{$\%$} \\
\hline Muy Baja & Litosol & 109402.3 & 8.1 \\
\hline Baja & $\begin{array}{c}\text { Luvisol, } \\
\text { Cambisol }\end{array}$ & 59511.9 & 4.4 \\
\hline Media & $\begin{array}{c}\text { Regosol, } \\
\text { Planosol, } \\
\text { Vertisol } \\
\text { Xerosol }\end{array}$ & 482026.2 & 35.9 \\
\hline Alta & Feozem & 693045.5 & 51.6 \\
\hline
\end{tabular}

(Fuente: elaboración propia, con base en la reclasificación de la cartografía de suelos de INEGI, 2007)

Tabla 3. Superficie de las distintas clases de pendiente en la zona de estudio.

\begin{tabular}{|c|c|c|c|}
\hline Categoría & Grados & $\begin{array}{c}\text { Superficie } \\
\text { ha }\end{array}$ & \% \\
\hline $\begin{array}{c}\text { Muy ligeramente } \\
\text { inclinadas }\end{array}$ & $<3$ & 508866.6 & 37.9 \\
\hline $\begin{array}{c}\text { Ligeramente } \\
\text { inclinadas }\end{array}$ & $3-5$ & 106234.0 & 7.9 \\
\hline $\begin{array}{c}\text { Medianamente } \\
\text { inclinadas }\end{array}$ & $5-10$ & 204849.6 & 15.2 \\
\hline $\begin{array}{c}\text { Fuertemente } \\
\text { inclinadas }\end{array}$ & $10-30$ & 428696.4 & 31.9 \\
\hline $\begin{array}{c}\text { Muy } \\
\text { Fuertemente } \\
\text { inclinadas }\end{array}$ & $>30$ & 95349.7 & 7.1 \\
\hline
\end{tabular}

(Fuente: elaboración propia, a partir de la reclasificación de Modelo Digital de Elevación del Terreno provisto por INEGI, 2013a)

Otras variables de tipo biofísico, como los ríos y escurrimientos se obtuvieron de la cartografía oficial de INEGI, pero se actualizaron mediante fotointerpretación a escala 1:50,000. La capa resultante se procesó mediante operadores de contexto espacial para obtener la distancia euclidiana a cada uno de los segmentos. 
Bravo Peña L. C., Torres Olave M. E., Alatorre Cejudo L. C., Castellanos Villegas A. E., Moreno Murrieta R.. L, Granados Olivas A., Uc Campos M., González León M., Wiebe Quintana L. C. (2017): "Áreas probables de degradación-deforestación de la cubierta vegetal en Chihuahua, México. Una exploración mediante regresión logística para el período 1985-2013", GeoFocus (Artículos), $n^{\circ}$ 20, p. 109-137. ISSN: 1578-5157 http://dx.doi.org/10.21138/GF.545

La representación espacial de las variables biofísicas empleadas en la regresión se observa en la Figura 2.
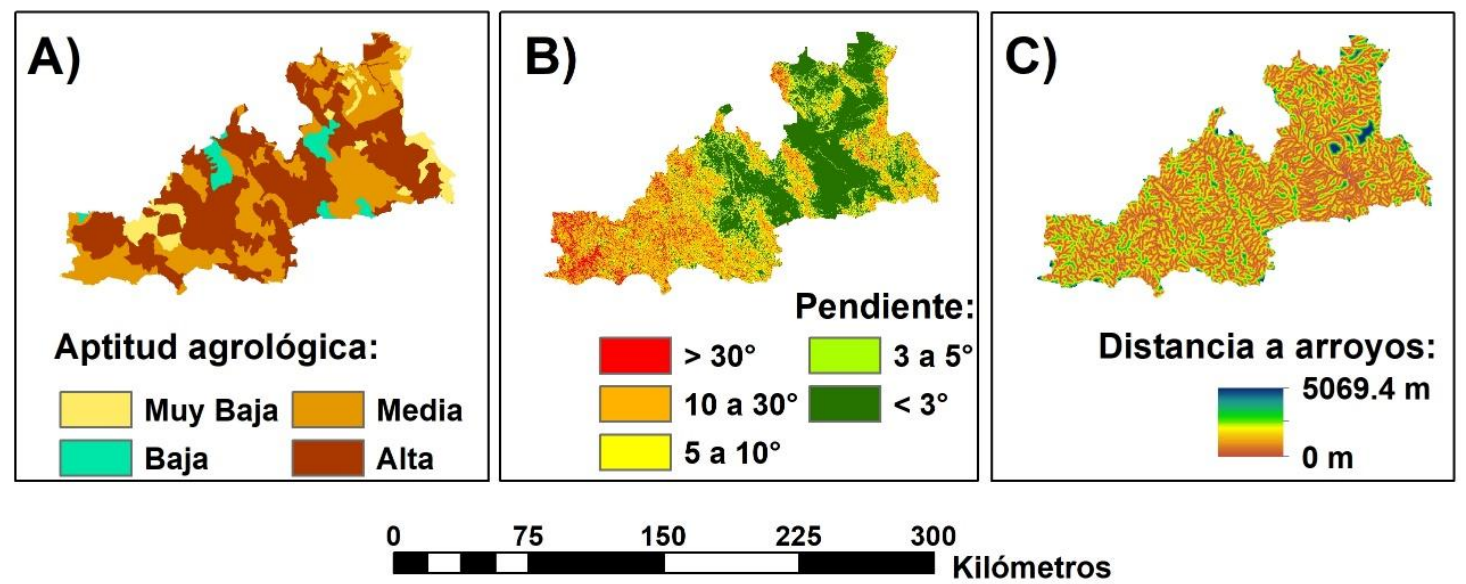

Figura 2. Insumos de carácter biofísico utilizados en la regresión logística: a) Aptitud agrológica del suelo, b) Pendiente del terreno, c) Distancia a arroyos y escurrimientos superficiales. (Fuente:

elaboración propia)

Por lo que respecta a variables de tipo socioeconómico, estas también se actualizaron o derivaron de información publicada, y se procesaron mediante operadores de contexto espacial (cálculo de distancias euclidianas), o bien se reclasificaron en rangos. En el primer caso pueden mencionarse las capas de carreteras y terracerías, polígonos agrícolas, localidades y pozos de extracción de agua subterránea; mientras que en el segundo caso se incluyen capas como tenencia de la tierra, marginación en el año de 1995, y cambios en la marginación durante el periodo 1995-2010. Las capas de Carreteras y caminos de terracerías, inicialmente en formato vectorial (4216685.2 y 1, 089,537.5 m lineales respectivamente), se obtuvieron de la cartografía de INEGI, pero se actualizaron mediante fotointerpretación y digitalización manual en un mosaico de ortofotos aéreas e imágenes Landsat correspondientes al periodo de análisis. A su vez, el mapa de polígonos agrícolas se obtuvo de aislar o seleccionar los 226 polígonos agrícolas de riego o temporal (332183.9 ha), que aparecen en la cartografía correspondiente a 1985.

Las localidades se generaron de un vector de puntos con las coordenadas de los centros de población urbanos y rurales, censados en el XII censo nacional de población y vivienda (INEGI, 2010). En este caso, a diferencia de lo realizado con el mapa de polígonos agrícolas, sólo se utilizaron las coordenadas puntuales de cada localidad, pues la región se distingue por la presencia y dispersión de numerosos caseríos y rancherías pobladas por indígenas Tarahumaras (Porras, 1999), cuyo tamaño -a menudo integrados por dos o tres viviendas- impidió su mapeo considerando el criterio de área mínima cartografiable y la escala a la que digitalizaron los mapas $(1: 250,000)$. La zona es habitada por 207,695 habitantes, distribuidos en 1,098 localidades (X a XII Censos Nacionales de Población y Vivienda, INEGI 1990, 2000 y 2010), pero solo cinco de estas $(0.5 \%$ del total de localidades) poseen población por arriba de 2500 personas. De hecho una sola localidad del área de estudio, Ciudad Cuauhtémoc (114,007 habitantes), concentra a más del $50 \%$ de la población total. Cabe decir en este sentido, que los polígonos de las localidades solo se consideraron en el cálculo de coberturas y en el análisis de cambios, pero no en el cálculo de distancias. 
Bravo Peña L. C., Torres Olave M. E., Alatorre Cejudo L. C., Castellanos Villegas A. E., Moreno Murrieta R.. L, Granados Olivas A., Uc Campos M., González León M., Wiebe Quintana L. C. (2017): "Áreas probables de degradación-deforestación de la cubierta vegetal en Chihuahua, México. Una exploración mediante regresión logística para el período 1985-2013", GeoFocus (Artículos), $n^{\circ}$ 20, p. 109-137. ISSN: 1578-5157 http://dx.doi.org/10.21138/GF.545

También los pozos agrícolas de extracción de agua subterránea se representaron mediante un mapa de distancia. Los pozos facilitan la conversión de los usos del suelo, de forestal a agropecuario, pues permiten irrigar nuevas tierras agrícolas, o la disponibilidad de agua para ranchos ganaderos. Las coordenadas de los pozos corresponden a 4360 concesiones de aprovechamiento de aguas nacionales existentes en el registro público de derechos de agua (REPDA, CONAGUA, 2015). Del total de pozos de extracción, el $62.8 \%$ de los pozos concesionados se destina exclusivamente al uso agrícola (2378 pozos), aunque es usual que los pozos restantes se utilicen para fines agropecuarios de forma intermitente. Esta razón justificó el cálculo de distancias a todos ellos.

Por lo que refiere a capas de información ordenadas en rangos o clases, se incluyeron la tenencia de la tierra, la marginación en el año 1995 y sus cambios en el periodo 1995-2010. En relación a tenencia de la tierra, distintos autores han mencionado la importancia de esta variable en la degradación de la cubierta vegetal, favoreciendo o evitando este proceso. En el caso de este trabajo, su mapa se obtuvo, del registro agrario nacional (RAN, 2012), y de un mapa de la propiedad de la tierra correspondiente al estado de Chihuahua digitalizado durante el desarrollo de este ejercicio a una escala de 1:100,000, con un error cartográfico máximo de 89 metros. La tenencia fue reclasificada en tres grandes categorías: 1) Propiedad ejidal que incluye terrenos ejidales (De propiedad social), 2) Propiedad privada, que incluye propiedad individual, y 3) Propiedad nacional, que se refiere básicamente a cuerpos de agua superficial, que en las leyes mexicanas pertenecen al Estado. Propiedad ejidal y propiedad privada se localizan en toda la región, pero la propiedad ejidal es frecuente hacia el oeste de la zona de estudio, donde existen la orografía más accidentada y las masas forestales más grandes de los cuatro municipios. La propiedad privada por el contrario, es muy frecuente hacia el este, donde las pendientes son más suaves, y hay mejores condiciones para la explotación agropecuaria del terreno. Las cantidades totales y proporciones se observan en la Tabla 4.

Tabla 4. Cobertura por tipo de propiedad de la tierra en la zona de estudio.

\begin{tabular}{|c|r|r|}
\hline $\begin{array}{c}\text { Tipo de } \\
\text { propiedad }\end{array}$ & $\begin{array}{c}\text { Cobertura } \\
\text { (ha) }\end{array}$ & Porcentaje \\
\hline Ejidal & 603973.3 & 45.3 \\
\hline Privada & 730611.6 & 54.7 \\
\hline TOTAL & 1334584.9 & 100 \\
\hline
\end{tabular}

(Fuente: elaboración propia, con base en los registros de RAN, 2012)

La representación espacial de las variables socioeconómicas a) Distancia a polígonos agrícolas de 1985, b) Distancia a carreteras, c) Distancia a caminos de terracería, d) Distancia a localidades, e) Distancia a pozos de extracción de agua subterránea, f) Tenencia de la tierra se observa en la Figura 3. 


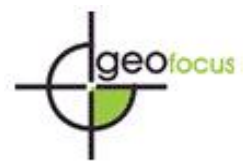

Bravo Peña L. C., Torres Olave M. E., Alatorre Cejudo L. C., Castellanos Villegas A. E., Moreno Murrieta R.. L, Granados Olivas A., Uc Campos M., González León M., Wiebe Quintana L. C. (2017): "Áreas probables de degradación-deforestación de la cubierta vegetal en Chihuahua, México. Una exploración mediante regresión logística para el período 1985-2013", GeoFocus (Artículos), $n^{\circ}$ 20, p. 109-137. ISSN: 1578-5157 http://dx.doi.org/10.21138/GF.545

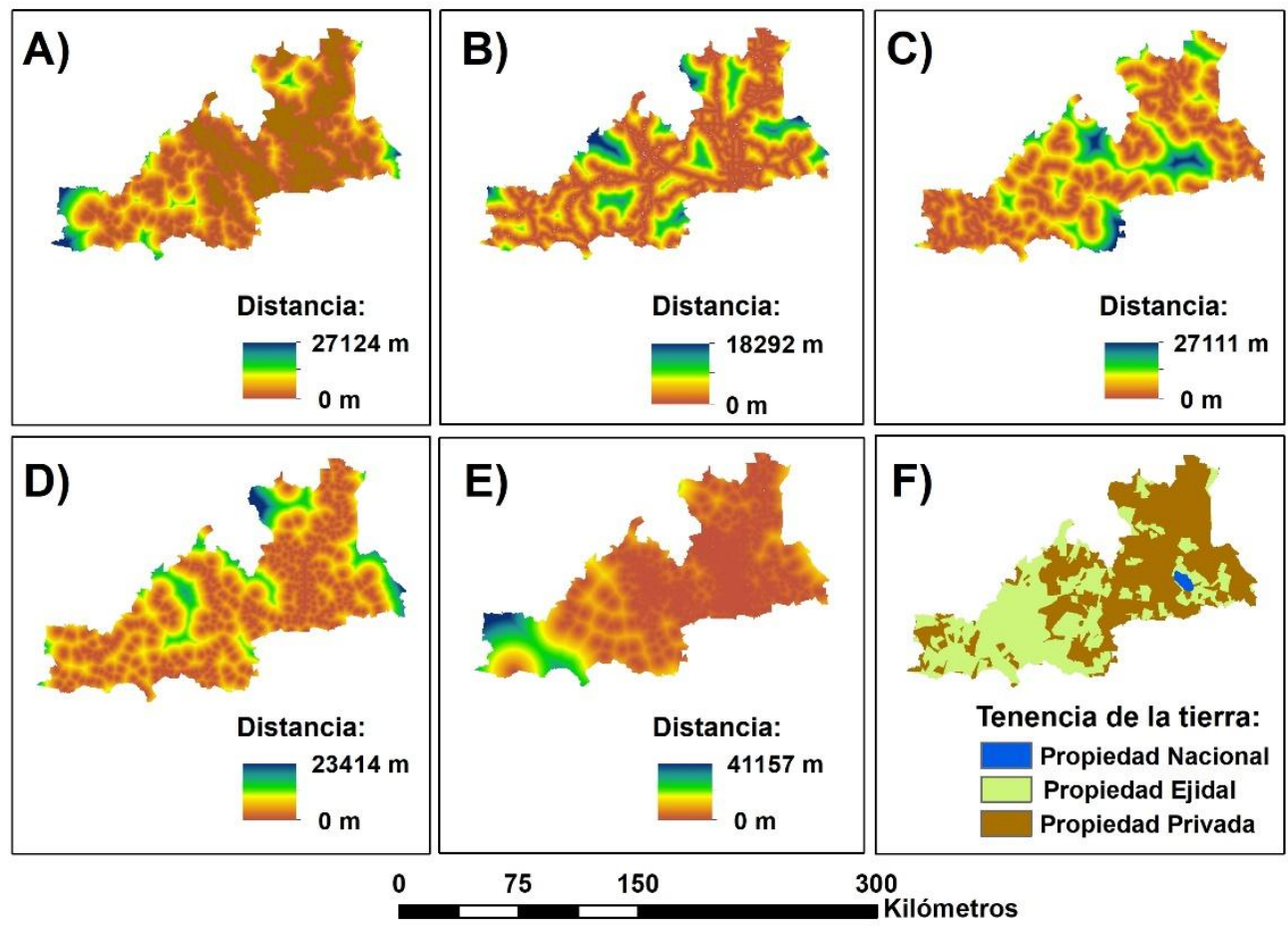

Figura 3. Variables socioeconómicas explicativas: a) Distancia a polígonos agrícolas de 1985, b) Distancia a carreteras, c) Distancia a caminos de terracería, d) Distancia a localidades, e) Distancia a pozos de extracción de agua subterránea, f) Tenencia de la tierra. (Fuente: elaboración propia)

Las dos capas restantes incluidas en el trabajo fueron marginación en el año de 1995 y los cambios en la marginación durante el periodo 1995-2010. Ambas representan espacialmente un indicador que mide la pobreza de forma multidimensional (en ingreso, educación y salud), que se ha relacionado previamente, con procesos de deforestación en el país (Morales-Barquero, 2015 y Pineda Jaimes et al., 2009). Aunque existe un desfase de 10 años respecto a la cartografía más antigua empleada en este trabajo, se incluyó la marginación de 1995 porque las mediciones de marginación en México iniciaron en ese año (CONABIO, 2001), y su importancia ha sido descrita por autores previos. Para la construcción del mapa de cambios, también se incluyó la medición más reciente correspondiente al año de 2010 (CONABIO, 2012). En las mediciones de 1995 y 2010, se determinó la marginación en 501 y 490 localidades de la zona de estudio (respectivamente), y sus resultados se integraron en este trabajo mediante la generación de superficies discretas obtenidas con el método de polígonos de Thiessen. Este método, que se ha utilizado con éxito en la geografía humana ( $\mathrm{Mu}, 2009)$, permitió obtener las superficies de marginación para cada año (Tabla 5). 
Bravo Peña L. C., Torres Olave M. E., Alatorre Cejudo L. C., Castellanos Villegas A. E., Moreno Murrieta R.. L, Granados Olivas A., Uc Campos M., González León M., Wiebe Quintana L. C. (2017): "Áreas probables de degradación-deforestación de la cubierta vegetal en Chihuahua, México. Una exploración mediante regresión logística para el período 1985-2013”, GeoFocus (Artículos), $n^{\circ}$ 20, p. 109-137. ISSN: 1578-5157 http://dx.doi.org/10.21138/GF.545

Tabla 5. Áreas observadas por grado de marginación en la zona de estudio en 1995.

\begin{tabular}{|c|c|r|c|r|}
\hline Marginación & $\begin{array}{c}\text { Área (ha) } \\
\mathbf{1 9 9 5}\end{array}$ & $\begin{array}{c}\text { Porcentaje } \\
\mathbf{1 9 9 5}\end{array}$ & $\begin{array}{c}\text { Área (ha) } \\
\mathbf{2 0 1 0}\end{array}$ & $\begin{array}{c}\text { Porcentaje } \\
\mathbf{2 0 1 0}\end{array}$ \\
\hline Muy Baja & 136963.4 & 10.2 & 278098.3 & 20.7 \\
\hline Baja & 292541.8 & 21.8 & 207474.7 & 15.4 \\
\hline Media & 310622.9 & 23.1 & 288276.7 & 21.4 \\
\hline Alta & 178920.6 & 13.3 & 352993.1 & 26.3 \\
\hline Muy Alta & 424947.7 & 31.6 & 217152.3 & 16.2 \\
\hline
\end{tabular}

(Fuente: elaboración propia, con base en los datos de CONABIO, 2001)

Con las capas de marginación correspondientes a 1995 y 2010, se realizó una operación de álgebra de mapas, a fin de obtener el comportamiento de esta variable entre ambos cortes temporales (Tabla 6):

Tabla 6. Clases de cambios en la categoría marginación durante el periodo 19952010.

\begin{tabular}{|c|c|c|c|}
\hline Clase & Cambios durante el periodo & $\begin{array}{l}\text { Superficie } \\
\text { (ha) }\end{array}$ & Porcentaje \\
\hline 1 & $\begin{array}{c}\text { Incremento de la marginación en } 4 \\
\text { categorías. }\end{array}$ & 458.7 & 0.03 \\
\hline 2 & $\begin{array}{l}\text { Incremento de la marginación en } 3 \\
\text { categorías. }\end{array}$ & 13255.6 & 1.0 \\
\hline 3 & $\begin{array}{c}\text { Incremento de la marginación en } 2 \\
\text { categorías. }\end{array}$ & 33863.3 & 2.5 \\
\hline 4 & $\begin{array}{c}\text { Incremento de la marginación en } 1 \\
\text { categoría. }\end{array}$ & 167169.7 & 12.4 \\
\hline 5 & Marginación sin cambios en el periodo & 592626.2 & 44.1 \\
\hline 6 & $\begin{array}{c}\text { Reducción de la marginación en } 1 \\
\text { categoría. }\end{array}$ & 415799.3 & 31.0 \\
\hline 7 & $\begin{array}{c}\text { Reducción de la marginación en } 2 \\
\text { categorías. }\end{array}$ & 73589.6 & 5.5 \\
\hline 8 & $\begin{array}{c}\text { Reducción de la marginación en } 3 \\
\text { categorías. }\end{array}$ & 36695.1 & 2.7 \\
\hline 9 & $\begin{array}{c}\text { Reducción de la marginación en } 4 \\
\text { categorías. }\end{array}$ & 10538.9 & 0.8 \\
\hline
\end{tabular}

La distribución espacial de la marginación en los años 1995 y 2010, así como el cambio de esta variable en el periodo de análisis, se aprecia en la figura 4. 
Bravo Peña L. C., Torres Olave M. E., Alatorre Cejudo L. C., Castellanos Villegas A. E., Moreno Murrieta R.. L, Granados Olivas A., Uc Campos M., González León M., Wiebe Quintana L. C. (2017): "Áreas probables de degradación-deforestación de la cubierta vegetal en Chihuahua, México. Una exploración mediante regresión logística para el período 1985-2013", GeoFocus (Artículos), $n^{\circ}$ 20, p. 109-137. ISSN: 1578-5157 http://dx.doi.org/10.21138/GF.545

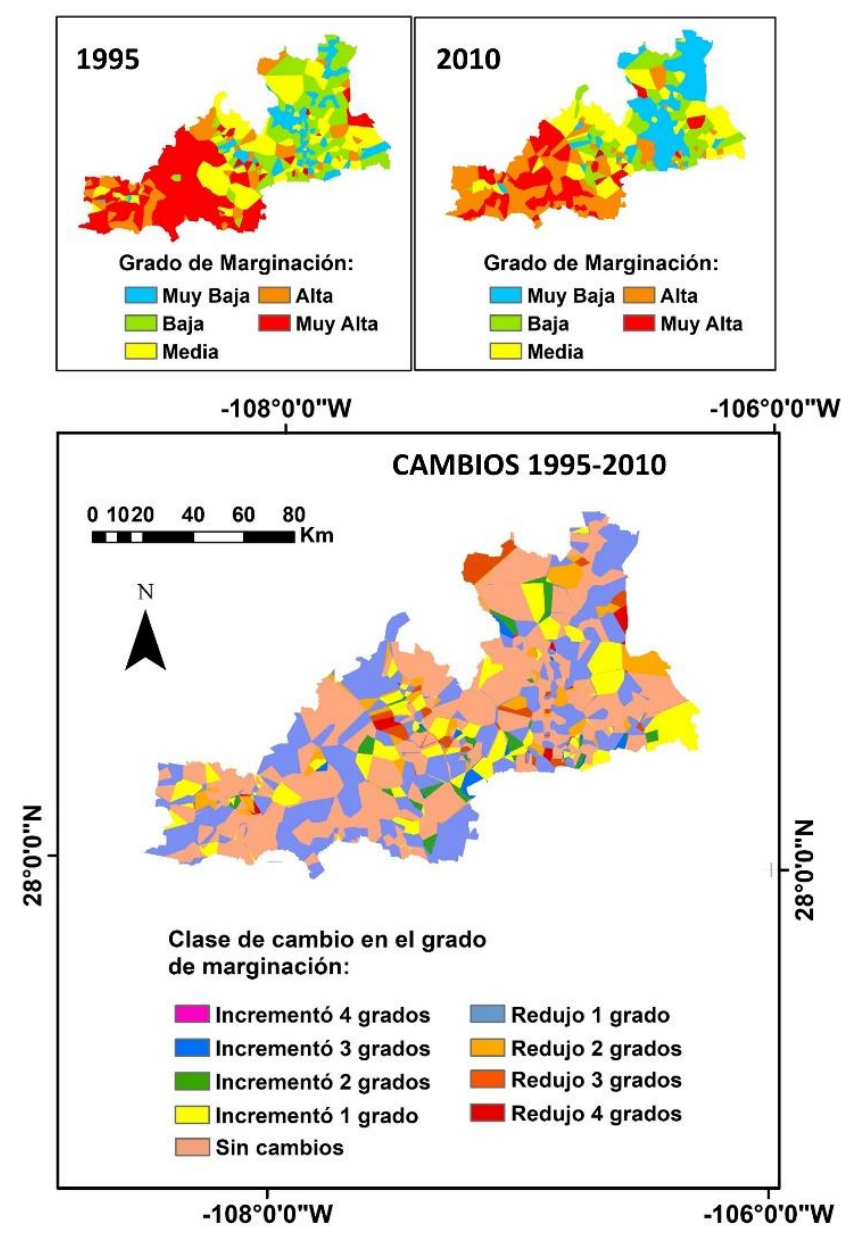

Figura 4. Marginación en 1995, 2010 y cambios en el mismo periodo.

(Fuente: elaboración propia, a partir de los datos de CONABIO, 2001 y 2012)

\subsection{Referentes teórico-estadísticos del método.}

En este trabajo se asume que la probabilidad de cambio de una celda correspondiente a cobertura forestal, a la clase degradado-deforestado, es función de los valores que toman las variables explicativas en la misma celda para cada uno de los mapas. Dicha función se puede representar mediante una curva logística, y su valor puede ser estimado con la base en la fórmula propuesta por Eastman (2012):

$$
P(y=1 \mathrm{I} X)=\frac{\exp \left(b 0+\sum b X\right)}{1+\operatorname{Exp}\left(b 0+\sum b X\right)}
$$

En esta ecuación, $\mathrm{P}$ es la probabilidad de que la variable dependiente tome el valor de $1, \mathrm{X}$ son las variables independientes o covariables $(\mathrm{X}=\mathrm{x} 1, \mathrm{x} 2, \mathrm{x} 3, \mathrm{x} 4, \mathrm{xn})$, b0 es la constante del modelo 
Bravo Peña L. C., Torres Olave M. E., Alatorre Cejudo L. C., Castellanos Villegas A. E., Moreno Murrieta R.. L, Granados Olivas A., Uc Campos M., González León M., Wiebe Quintana L. C. (2017): "Áreas probables de degradación-deforestación de la cubierta vegetal en Chihuahua, México. Una exploración mediante regresión logística para el período 1985-2013", GeoFocus (Artículos), $n^{\circ}$ 20, p. 109-137. ISSN: 1578-5157 http://dx.doi.org/10.21138/GF.545

o término independiente, y $\mathrm{b}$ representa los valores de las coeficientes a ser estimados para cada valor de $\mathrm{X}(\mathrm{B}=\mathrm{b} 1, \mathrm{~b} 2, \mathrm{~b} 3, \mathrm{~b} 4, \mathrm{bn})$, es decir, los coeficientes asociados a cada una de las variables predictoras, cuya magnitud es proporcional a la importancia de estas variables independientes para modelizar el comportamiento de variable de respuesta.

La ecuación anterior es de tipo exponencial, pero es transformada en una respuesta lineal mediante una transformación Logit, donde:

$$
\mathrm{P}=\operatorname{Ln}(\mathrm{P} /(1-\mathrm{P}))
$$

Que toma la forma de la ecuación de regresión lineal, donde Y=b0+bx

En este caso:

$$
\operatorname{Ln}(\mathrm{P} /(1-\mathrm{P}))=\mathrm{b} 0+\mathrm{b} 1 * \mathrm{x} 1+\mathrm{b} 2 * \mathrm{x} 2+\mathrm{b} 3 * \mathrm{x} 3+\mathrm{bn} * \mathrm{xn}+\text { error }
$$

Con la transformación anterior, la variable de salida crece linealmente conforme crece $\mathrm{x}$ (Rogerson, 2010). Asimismo, se vuelve una variable continua, teniendo valores que se ubican entre 0 y 1 . En el caso de este trabajo, dichos valores expresan la probabilidad del pixel a ser degradado-deforestado, y por lo tanto permiten identificar áreas con mayor susceptibilidad a sufrir este evento. Donde se observa cero la probabilidad de ser degradado-deforestado es nula, y donde se observa 1 , este proceso ya ocurrió.

\subsection{Contraste de bondad de ajuste del modelo de regresión logística.}

Posterior a la generación del modelo, se realizó una validación para evaluar su efectividad en la predicción de la ocurrencia de áreas degradadas-deforestadas. Esta validación incluye la determinación del valor de pseudo- $\mathrm{R}^{2}$ de McFadden, que es un indicador de la bondad de ajuste del modelo, aunque a diferencia del valor de $\mathrm{R}^{2}$ (coeficiente de determinación) en una regresión lineal convencional, no da una idea de la dispersión de los datos alrededor de la recta de regresión.

Pseudo- $\mathrm{R}^{2}$ de McFadden no se calcula ni se entiende como $\mathrm{R}^{2}$ en una regresión lineal convencional. En una RLM se requieren valores de $\mathrm{R}^{2}$ muy cercanos a 1 para decir que hubo buen ajuste, y buena capacidad predictiva (Rogerson, 2010), pero en el caso de pseudo- $\mathrm{R}^{2}$ de McFadden, generalmente se esperan valores bajos. Esto es así, porque la variable de respuesta con la que se construye el modelo tiene un comportamiento binario o dicotómico. Debido a esto, se asume que un buen modelo de regresión logística arroja valores de pseudo- $\mathrm{R}^{2}$ de McFadden mayores a 0.2 (Clark y Hosking, 1986; Wilson, Newton, Echeverría, Weston, \& Burgman, 2005).

En el caso de este trabajo, el valor obtenido de pseudo- $\mathrm{R}^{2}$ de McFadden fue el primer criterio de validación, pero adicionalmente se compararon las predicciones espaciales de áreas deforestadas, con los valores reales de ocurrencia espacial de las celdas deforestadas. Esta comparación se realizó mediante la curva ROC (Receiver Operating Characteristic), que compara la tasa de clasificaciones correctas respecto al total predicho, y observa los intervalos de acierto como criterio de calidad del modelo generado. De acuerdo a los referentes de Hall \& Hyndam (2003), y Hall, Hydam y Fan (2004), si el valor de ROC se ubica entre 0.97 y 1, el modelo ajusta de forma excelente; si se encuentra entre 0.97 y 0.90 tiene muy buen ajuste, si arroja 0.90 y 0.75 
Bravo Peña L. C., Torres Olave M. E., Alatorre Cejudo L. C., Castellanos Villegas A. E., Moreno Murrieta R.. L, Granados Olivas A., Uc Campos M., González León M., Wiebe Quintana L. C. (2017): "Áreas probables de degradación-deforestación de la cubierta vegetal en Chihuahua, México. Una exploración mediante regresión logística para el período 1985-2013", GeoFocus (Artículos), $n^{\circ}$ 20, p. 109-137. ISSN: 1578-5157 http://dx.doi.org/10.21138/GF.545

presenta un buen ajuste, mientras que presenta un ajuste regular si obtiene entre 0.75 y 0.60 . En el caso extremo, se observa un mal juste del modelo cuando este presenta valores inferiores a 0.60 .

\subsection{Significancia de los coeficientes de cada co-variable.}

Se determinó la significancia de los coeficientes correspondientes a cada variable, generados en la RLO, con un análisis de contrastes condicionales de razón de verosimilitud. Este análisis contrasta un modelo completo que incluye a todas las variables, con modelos parciales que se obtienen al eliminar de forma aislada cada una de las covariables que integran el modelo completo. En cada eliminación, la ausencia de significación muestra que el modelo sin la covariable de $\mathrm{X}$ correspondiente no empeora respecto al modelo completo, y permite suponer que es irrelevante para la ocurrencia de la variable Y. Tomando en cuenta el principio de parsimonia (Occam's razor), el modelo resultante final fue aquel donde se eliminan las variables que no aportan nada al modelo.

El estadístico de razón de verosimilitudes se obtiene con la fórmula

$\mathrm{ERV}=-2 \log \mathrm{L} 0--2 \log ($ likelihood $)$.

En esta ecuación, $2 \log (\mathrm{L} 0)$ es el valor de la función de verosimilitud si todos los coeficientes excepto el valor de intercepción valen 0 , mientras que -2log (Likelihood) es el valor de la función de verosimilitud para el modelo completo (Eastman, 2012). ERV tiene una distribución Chi cuadrada, y permite contrastar la hipótesis nula de que los coeficientes individuales de las variables explicativas, excepto el valor b de intercepción son iguales a cero, lo que indicaría que dichas variables no son relevantes para predecir el fenómeno modelado. Puesto que ERV sigue una distribución Chi cuadrada, se rechaza la hipótesis nula si dicho valor es mayor que un valor de Chi cuadrada obtenido en tablas, al nivel de significancia elegido ( $\mathrm{P}<$ 0.05 ) con los grados de libertad de la muestra, que se contabilizan como el número de variables explicativas a incluir. En este caso el número de grados de libertad fue 11, porque se incluyeron 11 variables explicativas.

Cabe decir que el método empleado en este trabajo para determinar las variables explicativas a incluir, se apega a la lógica del procedimiento de selección por pasos (stepwise) hacia atrás (backward), empleado con frecuencia en distintos paquetes estadísticos durante el procedimiento de regresión. En este caso, al igual que en stepwise backward, se incluyen todas las variables independientes en un primer ensayo, y posteriormente se procede a eliminar aquellas variables que siendo excluidas en los modelos parciales de regresión, no son significantes en el modelo completo. La diferencia más importante entre ambos procedimientos radica en el estadístico de prueba: Chi cuadrada en el análisis de contrastes condicionales (Eastman, 2012), y el estadístico F en stpewise backward (Pardo y Ruíz, 2002).

\section{Resultados y Discusión.}

3.1. Cambios de cobertura durante el periodo de medición (1985-2013).

Se observaron diez clases de cubierta vegetal y uso del suelo en el área de estudio (Figura $5)$. 
Bravo Peña L. C., Torres Olave M. E., Alatorre Cejudo L. C., Castellanos Villegas A. E., Moreno Murrieta R.. L, Granados Olivas A., Uc Campos M., González León M., Wiebe Quintana L. C. (2017): "Áreas probables de degradación-deforestación de la cubierta vegetal en Chihuahua, México. Una exploración mediante regresión logística para el período 1985-2013", GeoFocus (Artículos), $n^{\circ}$ 20, p. 109-137. ISSN: 1578-5157 http://dx.doi.org/10.21138/GF.545
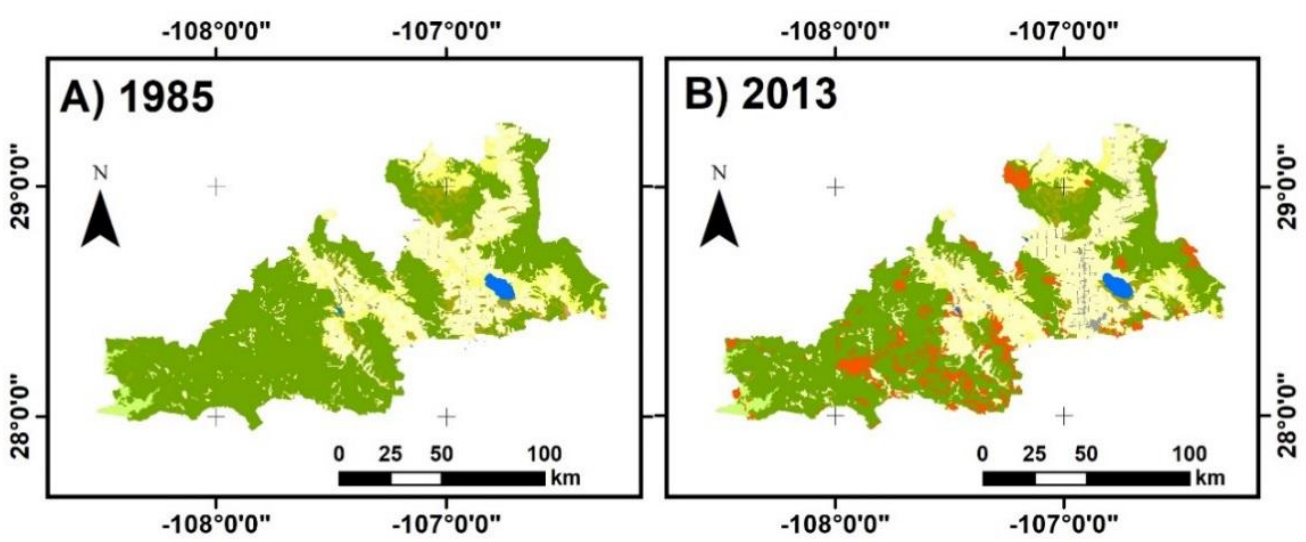

Simbología

\begin{tabular}{|c|c|}
\hline Agricultura & Cuerpo de Agua \\
\hline Asentamiento Humano & Sin Vegetación \\
\hline Bosque* & Matorral Xerófilo* \\
\hline
\end{tabular}

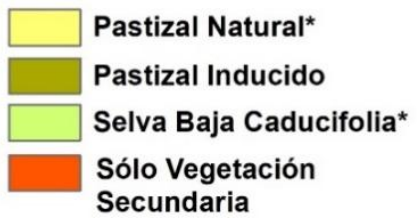

Figura 5. Usos del Suelo y Vegetación en el área de estudio. (Fuente: elaboración propia, con la información de Uso del Suelo y Vegetación de INEGI Series I y V.

* Coberturas compuestas por vegetación primaria, aunque pueden tener de forma marginal elementos de vegetación secundaria)

Los cambios anteriores, contabilizados de forma neta por cobertura, revelan que las coberturas naturales han perdido importantes superficies, mientras que las coberturas antropogénicas han experimentado una ganancia considerable (Tabla 7).

Tabla 7. Cambio neto entre categorías en el periodo 1985-2013.

\begin{tabular}{|c|c|c|c|}
\hline Categoría & Área en 1985 (ha) & $\begin{array}{l}\begin{array}{l}\text { Área en } \\
\text { (ha) }\end{array} \\
\end{array}$ & Cambio neto (ha) \\
\hline Bosque* & $830,597.8$ & $695,682.2$ & $-134,915.6$ \\
\hline Matorral Xerófilo* & $1,280.5$ & 396.1 & -884.4 \\
\hline Pastizal Natural* & $94,554.4$ & $69,499.6$ & $-25,054.8$ \\
\hline Selva Baja Caducifolia* & $18,581.1$ & $17,078.9$ & $-1,502.2$ \\
\hline Cuerpo de Agua & $10,368.8$ & $11,013.7$ & 644.9 \\
\hline Sin Vegetación & 109.5 & 876.7 & 767.2 \\
\hline Agricultura & $329,159.7$ & $378,082.1$ & $48,922.8$ \\
\hline Asentamiento Humano & $4,732.7$ & 18,307 & $13,574.3$ \\
\hline Pastizal Inducido & $54,602.2$ & $45,423.5$ & $-9,178.7$ \\
\hline $\begin{array}{ll}\text { Sólo } & \text { vegetación } \\
\text { secundaria } & \end{array}$ & $\begin{array}{r}\text { No se reporta como } \\
\text { clase }\end{array}$ & $107,626.5$ & $107,626.5$ \\
\hline
\end{tabular}

(Fuente: elaboración propia con los datos de los inventarios I y $\mathrm{V}$ de uso del suelo y vegetación de

INEGI. *Se trata de clases con vegetación primaria dominante, aunque pueden tener elementos de vegetación secundaria marginal.)

Destaca la pérdida o degradación severa experimentada por la clase bosque, de más de 134,915.6 ha; le sigue en importancia la clase pastizal natural, que ha perdido o degradado $25,054.8$ ha. Por lo que se refiere a las clases antropogénicas, la clase agricultura ha ganado una 
Bravo Peña L. C., Torres Olave M. E., Alatorre Cejudo L. C., Castellanos Villegas A. E., Moreno Murrieta R.. L, Granados Olivas A., Uc Campos M., González León M., Wiebe Quintana L. C. (2017): "Áreas probables de degradación-deforestación de la cubierta vegetal en Chihuahua, México. Una exploración mediante regresión logística para el período 1985-2013", GeoFocus (Artículos), $n^{\circ}$ 20, p. 109-137. ISSN: 1578-5157 http://dx.doi.org/10.21138/GF.545

superficie considerable $(48,922.8$ ha), pero la clase que más ha incrementado corresponde a sólo vegetación secundaria, probablemente por la degradación severa de las coberturas primarias naturales, que estaban en buen estado de conservación hacia 1985. Todos estos cambios, agrupados en las grandes categorías Natural y Antropogénico, revelan cambios netos importantes de una categoría a otra durante el periodo de referencia (Tabla 8 y Figura 6).

Tabla 8. Cambio Neto en las categorías Natural y Antropogénico en los años de referencia.

\begin{tabular}{|c|c|c|c|}
\hline Clase & Categoría & Superficie (ha) 1985 & Superficie (ha) 2013 \\
\hline Bosque* & \multirow{5}{*}{ Natural } & \multirow{5}{*}{955382.6} & \multirow{5}{*}{793670.5} \\
\hline Matorral Xerófilo* & & & \\
\hline Pastizal Natural* & & & \\
\hline Selva Baja Caducifolia* & & & \\
\hline Cuerpo de Agua & & & \\
\hline Agricultura & \multirow{5}{*}{ Antropogénico } & \multirow{5}{*}{388603.7} & \multirow{5}{*}{550315.8} \\
\hline Asentamiento Humano & & & \\
\hline Pastizal Inducido & & & \\
\hline Vegetación secundaria & & & \\
\hline Sin Vegetación & & & \\
\hline
\end{tabular}

(Fuente: elaboración propia, las áreas se obtuvieron a partir de la cartografía de Uso del Suelo y

Vegetación de INEGI, Inventarios 1 y V. * Vegetación primaria dominante, que presenta vegetación secundaria marginal)

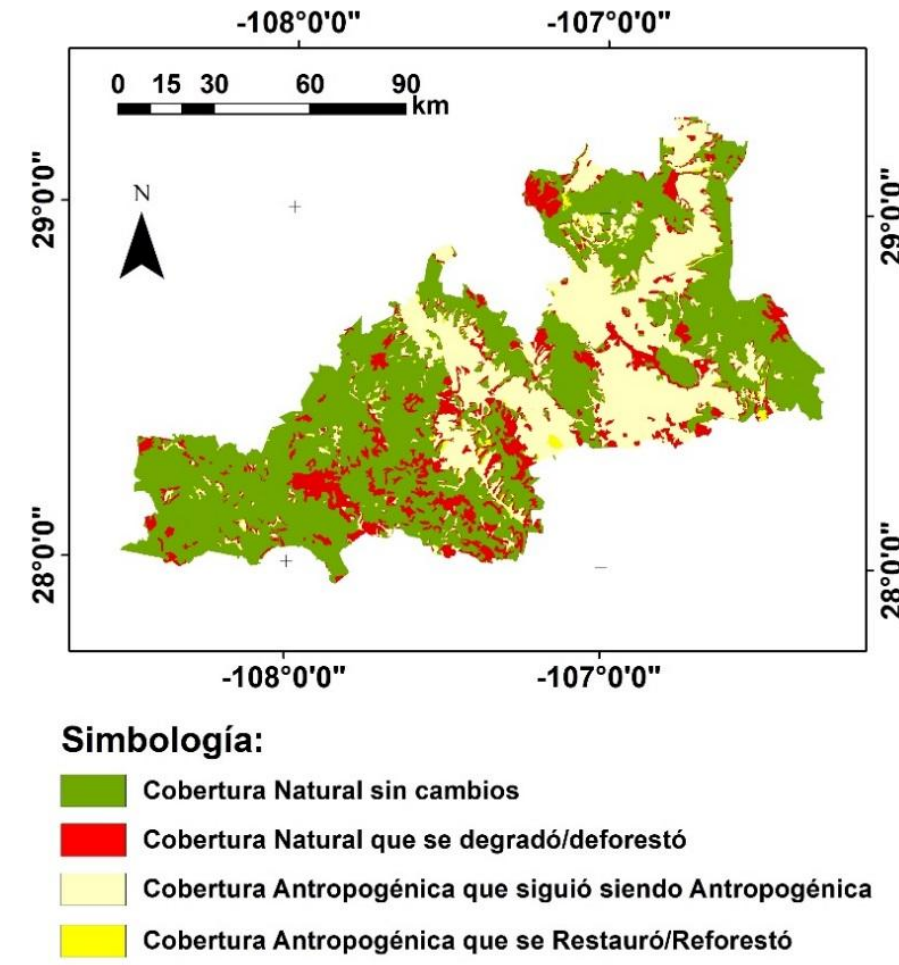

Figura 6. Cambios de cubierta vegetal y uso del suelo observados en la zona de estudio. (Fuente: elaboración propia, con la información de Uso del Suelo y Vegetación de INEGI, Series I y V) 
Bravo Peña L. C., Torres Olave M. E., Alatorre Cejudo L. C., Castellanos Villegas A. E., Moreno Murrieta R.. L, Granados Olivas A., Uc Campos M., González León M., Wiebe Quintana L. C. (2017): "Áreas probables de degradación-deforestación de la cubierta vegetal en Chihuahua, México. Una exploración mediante regresión logística para el período 1985-2013", GeoFocus (Artículos), $n^{\circ}$ 20, p. 109-137. ISSN: 1578-5157 http://dx.doi.org/10.21138/GF.545

Las transiciones entre las clases Natural-Antropogénico se aprecian más claramente en la matriz de tabulación cruzada (Tabla 9). Esta matriz consiste en una tabla de doble entrada, que muestra al final de cada columna la superficie de las clases natural y antropogénico en 1985, y al final de cada renglón las superficies en 2013 (Pontius et al., 2004:252). En la diagonal que va de la esquina superior izquierda a la esquina inferior derecha, se representan las superficies por cada categoría que permanecieron sin modificación de cobertura, mientras que los valores fuera de esta diagonal representan los cambios de cobertura entre 1985 y 2013: pérdidas de la clase que pasaron a otras categorías si están en las columnas, o ganancias de la clase, provenientes de otras categorías, si están en los renglones.

Tabla 9. Matriz de Tabulación Cruzada para el periodo de referencia en la zona de estudio.

\begin{tabular}{|c|c|c|c|}
\hline & $\begin{array}{c}\text { Natural } \\
\text { (ha) }\end{array}$ & $\begin{array}{c}\text { Antropogénico } \\
\text { (ha) }\end{array}$ & $\begin{array}{c}\text { Total 2013 } \\
\text { (ha) }\end{array}$ \\
\hline Natural & $773,065.6$ & $20,604.6$ & $793,670.5$ \\
\hline Antropogénico & 182,317 & $367,998.8$ & $550,315.8$ \\
\hline $\begin{array}{c}\text { Total 1985 } \\
\text { (ha) }\end{array}$ & $955,382.6$ & $388,603.4$ & $1,384,497.4$ \\
\hline
\end{tabular}

(Fuente: elaboración propia, con la información reclasificada de Uso del Suelo y Vegetación de INEGI, Series I y V; Los decimales en la sumatoria final pueden no corresponder por el redondeo)

Para entender mejor la dinámica espacio-temporal anterior, se ejemplificará con la cobertura natural. Esta tuvo 955382.6 ha en el año 1985 (final de la columna natural), mientras que presentó 793670.5 ha en el año 2013 (final del renglón natural). Durante este periodo, solo se conservaron 773065.6 ha (intersección columna renglón natural), mientras que se perdieron 182317 ha en el mismo lapso de tiempo. Esta superficie, compuesta básicamente de vegetación primaria con poca evidencia de disturbio, se degradó severamente o deforestó, sumándose a las coberturas antropizadas existentes al año 2013. Por otro lado, la cobertura natural ganó 20604.6 ha de áreas con cubierta antropogénica que probablemente se abandonaron, y se regeneraron por efecto de la sucesión ecológica. Por lo que se refiere a la clase antropogénico, esta presentó 388603 ha en 1985, y 550315.8 ha en 2013. De estas últimas, la superficie que se conservó sin cambios de un año a otro fue de 367998.8 , mientras que 182317 ha es la superficie que se añadió a la clase por deforestación de la cobertura natural. Asimismo, como se indicó previamente, esta clase con cobertura antropogénica perdió 20604.6 ha, de áreas que probablemente se abandonaron, y se regeneraron por efecto de la sucesión ecológica, pasando a la clase natural.

\subsection{Resultados de la Regresión Logística.}

Con las variables descritas en la sección anterior, se realizó el ensayo de regresión logística. Los resultados de regresión obtenidos mostraron que es posible predecir con precisión la distribución de superficies susceptibles de deforestación en el área de estudio. El valor de Pseudo $\mathrm{R}^{2}$ resultante de regresar los procesos de deforestación contra las variables explicativas fue de 0.24 , que es muy aceptable de acuerdo a los parámetros del método. 
Bravo Peña L. C., Torres Olave M. E., Alatorre Cejudo L. C., Castellanos Villegas A. E., Moreno Murrieta R.. L, Granados Olivas A., Uc Campos M., González León M., Wiebe Quintana L. C. (2017): "Áreas probables de degradación-deforestación de la cubierta vegetal en Chihuahua, México. Una exploración mediante regresión logística para el período 1985-2013", GeoFocus (Artículos), $n^{\circ}$ 20, p. 109-137. ISSN: 1578-5157 http://dx.doi.org/10.21138/GF.545

Vale notar que a diferencia de lo esperado en una regresión lineal convencional, donde $\mathrm{R}^{2}$ o el coeficiente de determinación debe tener valores cercanos a 1 para que la regresión tenga un elevado poder predictivo (Rogerson, 2010), en el caso de Pseudo $\mathrm{R}^{2}$ de McFadden se esperan valores bajos, porque la variable de respuesta con la que se construye el modelo tiene un comportamiento binario o dicotómico. Por esta razón se asume que un buen modelo de regresión logística arroja valores de Pseudo $\mathrm{R}^{2}$ de McFadden mayores a 0.2 (Clark y Hosking, 1986; Wilson, Newton, Echeverría, Weston, \& Burgman, 2005), por lo que puede decirse que el valor alcanzado en este ejercicio fue bastante bueno (Pseudo $R^{2}$ de 0.24 ). Este sugiere que el modelo generado permite predecir con un nivel aceptable las áreas susceptibles de ser deforestadas.

Por lo que respecta al estadístico de razón de verosimilitudes (ERV=-2logL0--2log (likelihood), este arrojó un valor de Chi cuadrado de 1496 402.7, superando ampliamente el valor de $\mathrm{Chi}^{2}$ de tablas al 0.05 de significancia para 11 grados de libertad (once variables explicativas), que fue de 19675 . Dicho resultado indica que todas las variables explicativas incluidas en el trabajo contribuyen a explicar los procesos de degradación-deforestación en la zona de estudio, aunque por el valor obtenido en los coeficientes, cuatro de estas variables (tenencia de la tierra, marginación, cambio en la marginación, aptitud agrológica del suelo y pendiente), representan los factores de mayor importancia para predecir las áreas susceptibles de ser degradadas-deforestadas. Las siete variables restantes fueron significantes en términos matemáticos, pero con un peso tan pequeño, que casi son irrelevantes (Tabla 10).

Tabla 10. Coeficientes obtenidos para cada variable.

\begin{tabular}{|c|c|r|}
\hline Variable & Coeficiente & $\begin{array}{c}\text { Valor } \\
\text { estandarizado }\end{array}$ \\
\hline No aplica & Constante & -5.60 \\
\hline Tenencia de la tierra & Te & 0.45 \\
\hline $\begin{array}{c}\text { Marginación en el año } \\
1995\end{array}$ & Marg95 & 0.28 \\
\hline $\begin{array}{c}\text { Cambios de la } \\
\text { marginación 1995-2010 }\end{array}$ & CMarg95-2010 & 0.21 \\
\hline $\begin{array}{c}\text { Aptitud agrológica del } \\
\text { suelo }\end{array}$ & CEd & 0.18 \\
\hline Pendiente & Pe & 0.13 \\
\hline $\begin{array}{c}\text { Distancia a polígonos } \\
\text { agrícolas }\end{array}$ & DPA & 0.00 \\
\hline Distancia a carreteras & DCa & $-0.00^{*}$ \\
\hline $\begin{array}{c}\text { Distancia a caminos de } \\
\text { terracería }\end{array}$ & DBr & $0.00^{*}$ \\
\hline Distancia a localidades & DLoc & $0.00^{*}$ \\
\hline $\begin{array}{c}\text { Distancia a pozos de } \\
\text { extracción de agua } \\
\text { subterránea }\end{array}$ & DRepda & $-0.00^{*}$ \\
\hline Distancia escurrimientos & DEsc & $0.00^{*}$ \\
\hline
\end{tabular}

* Se refiere a valores muy pequeños del coeficiente, de hasta cinco ceros a la derecha del punto, significantes en términos matemáticos de acuerdo al método, pero irrelevantes al proceso. 
Bravo Peña L. C., Torres Olave M. E., Alatorre Cejudo L. C., Castellanos Villegas A. E., Moreno Murrieta R.. L, Granados Olivas A., Uc Campos M., González León M., Wiebe Quintana L. C. (2017): "Áreas probables de degradación-deforestación de la cubierta vegetal en Chihuahua, México. Una exploración mediante regresión logística para el período 1985-2013", GeoFocus (Artículos), $n^{\circ}$ 20, p. 109-137. ISSN: 1578-5157 http://dx.doi.org/10.21138/GF.545

En la tabla anterior, los parámetros estandarizados muestran que las variables que tienen un peso mayor para predecir las superficies susceptibles de degradación-deforestación son Tenencia de la tierra (0.45), marginación en el año 1995 (0.28), cambios de marginación en el periodo 95-2010 (0.21), aptitud agrológica del suelo (0.18) y pendiente (0.13). El resto de las otras variables tuvo un peso significativamente menor (hasta cinco ceros a la derecha del punto), lo que sugiere que no son determinantes. En relación a esto, para entender la relación que se observa entre cada variable explicativa y la degradación- deforestación, es necesario recordar que un coeficiente positivo indica una relación positiva entre la variable explicativa y la variable predicha, es decir, que los valores de la segunda crecen al tiempo que ocurre lo mismo con los valores de la primera; mientras que un coeficiente negativo indica que la variable predicha decrece, mientras que la variable explicativa aumenta.

Con base en lo anterior, si se observa la relación entre áreas degradadas-deforestadas y tenencia de la tierra $(\mathrm{Te})$, la probabilidad de degradación-deforestación tiende a ser mayor en áreas de propiedad privada respecto a las áreas de propiedad social en el periodo evaluado. Este tipo de relación positiva igualmente se observa frente a las variables Marginación en el año 1995 (Marg95) y cambios en la marginación durante el periodo 1995-2010 (CMarg95-2010); lo que muestra que en la medida que la marginación se incrementa, la probabilidad de degradación-deforestación también se incrementa. La relación positiva también aparece frente a la aptitud agrológica del suelo (Ced), lo que sugiere mayor probabilidad de degradacióndeforestación en zonas donde el suelo presenta condiciones relativamente apropiadas para el desarrollo de cultivos agrícolas. Finalmente cabe señalar la relación que se observa con la variable pendiente del terreno $(\mathrm{Pe})$, pues en la medida que la pendiente muestra valores más pronunciados se observa una relación positiva con la probabilidad de degradacióndeforestación, lo que probablemente se vincula con el hecho de que las actividades causantes de este proceso (agricultura, apertura de pastizales ganaderos, extracción forestal, etc.) se desarrollan en áreas con condiciones físicas, básicamente pendiente, cada vez menos idóneas. Si esto ocurre, es de esperarse que el impacto ambiental del proceso de degradación-deforestación se amplifique, pues la pérdida de cubierta vegetal en áreas de elevada pendiente, significará además de la pérdida en biodiversidad, mayor probabilidad de erosión y la pérdida de suelo.

La distribución espacial de la probabilidad de deforestación se muestra en la figura 7. En la literatura se indica que valores de probabilidad superiores a 0.5 en la técnica de RLO pueden considerarse como altas probabilidades (Zheng \& Fan, 2015). Este umbral sugiere que en la zona son pocas las áreas susceptibles de ser deforestadas, ubicándose la mayor parte del área de estudio, en un umbral de bajo riesgo. No obstante eso, debe decirse que el riesgo puede modificarse si se modifican las condiciones socioeconómicas prevalecientes al momento de hacer este estudio. Cambios en el índice de marginación en los años subsecuentes, por ejemplo, podrían modificar el mapa, y poner en riesgo zonas que no se han detectado al momento de realizar este ejercicio. En el mismo sentido, factores no contemplados en este trabajo, como variaciones en el precio de los productos agrícolas, políticas gubernamentales de subsidio a la producción agropecuaria (Klepeis \& Vance, 2003), o incluso la actuación de las bandas dedicadas al narcotráfico y otros ilícitos, pueden cambiar la localización espacial de las áreas susceptibles a degradarse-deforestarse. Dichas posibilidades obligan al monitoreo constante de este fenómeno, pues probablemente el tipo de interacción entre las variables explicativas y la degradación se modifique, modificando también el signo y la magnitud de los coeficientes. 
Bravo Peña L. C., Torres Olave M. E., Alatorre Cejudo L. C., Castellanos Villegas A. E., Moreno Murrieta R.. L, Granados Olivas A., Uc Campos M., González León M., Wiebe Quintana L. C. (2017): "Áreas probables de degradación-deforestación de la cubierta vegetal en Chihuahua, México. Una exploración mediante regresión logística para el período 1985-2013", GeoFocus (Artículos), $n^{\circ}$ 20, p. 109-137. ISSN: 1578-5157 http://dx.doi.org/10.21138/GF.545

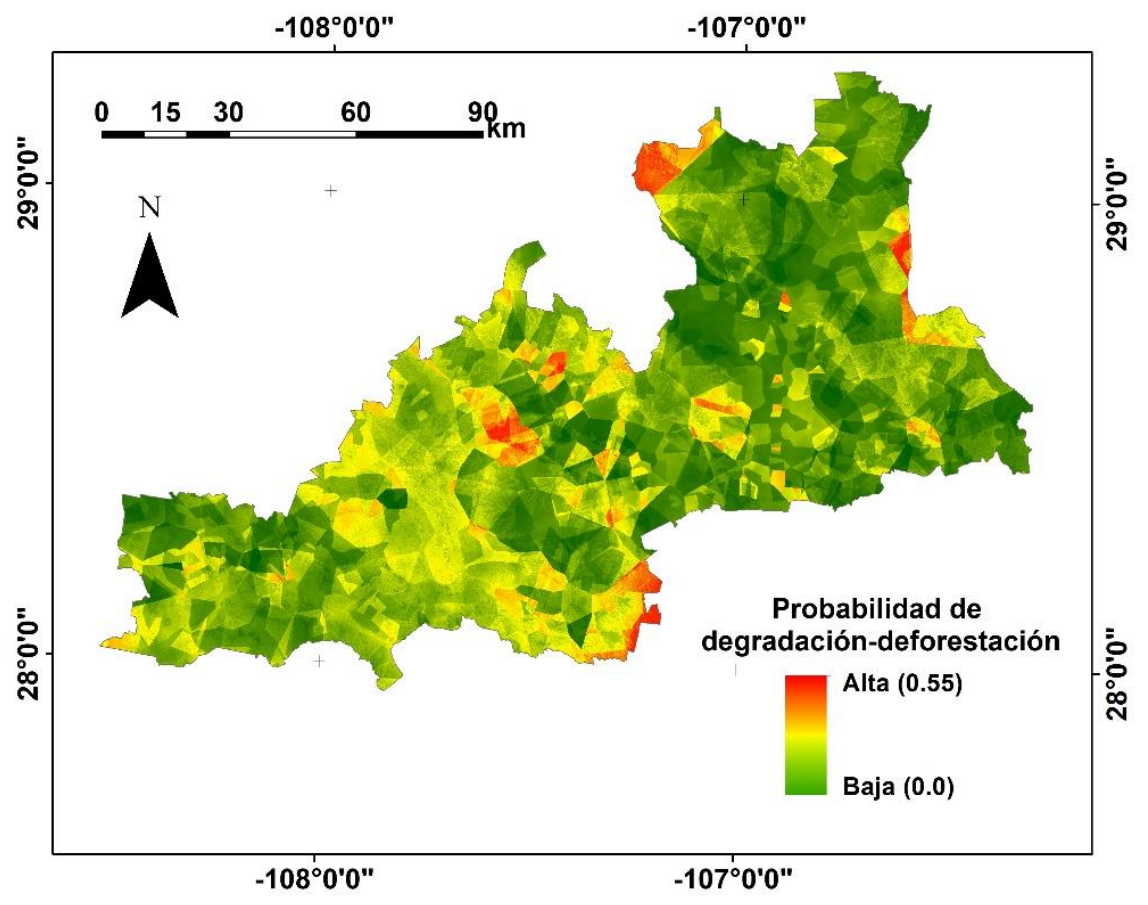

Figura 7. Probabilidad de degradación-deforestación en el área de estudio.

(Fuente: elaboración propia)

Por lo que se refiere al ajuste entre las predicciones y la realidad, ROC tuvo un valor de 0.85 , que indica un buen ajuste entre ambas variables, indicando que las áreas predichas por el modelo de regresión superan ampliamente los resultados que se habrían generado sólo por efecto del azar. Estos valores respaldan el coeficiente de Pseudo $\mathrm{R}^{2}$ obtenido, e indican que la predicción es confiable. De acuerdo a ella, es posible identificar donde hay más probabilidad de degradación-deforestación, dispersándose estas zonas en toda el área de estudio, pero predominando en el centro de la misma y en áreas muy localizadas al norte y al este. Se trata básicamente de áreas donde se observa una orografía accidentada, con frecuencia un elevado aislamiento de localidades, y un conjunto de condiciones físicas que favorecen la pérdida o degradación de la cubierta vegetal. Las condiciones socioeconómicas prevalecientes, en conjunto con los factores físicos, propician los cambios de cubierta vegetal y uso de suelo, de forestal a no forestal observados en este trabajo.

Los resultados obtenidos coinciden parcialmente con otros autores que han abordado el estudio de la deforestación en México mediante técnicas de regresión logística o multivariante (Morales-Barquero et al., 2015 y Pineda Jaimes et al., 2009), quienes han encontrado que son los factores socio-demográficos quienes parecen contribuir con más fuerza a este proceso. Morales-Barquero et al (2015), por ejemplo, en su estudio de la deforestación en una cuenca del occidente de México cubierta por bosque tropical seco, encontró que las variables marginación y proporción de área de bosque respecto al tamaño de la población, fueron los factores de mayor peso para explicar la pérdida. Por lo que respecta al estudio de Pineda Jaimes et al. (2009), en su 
Bravo Peña L. C., Torres Olave M. E., Alatorre Cejudo L. C., Castellanos Villegas A. E., Moreno Murrieta R.. L, Granados Olivas A., Uc Campos M., González León M., Wiebe Quintana L. C. (2017): "Áreas probables de degradación-deforestación de la cubierta vegetal en Chihuahua, México. Una exploración mediante regresión logística para el período 1985-2013", GeoFocus (Artículos), $n^{\circ}$ 20, p. 109-137. ISSN: 1578-5157 http://dx.doi.org/10.21138/GF.545

exploración de la pérdida de Bosques en el Estado de México, este encuentra que es el grado de analfabetismo (una variable muy relacionada con marginación), el factor que parece contribuir más a la disminución de la cobertura de bosques en su zona de estudio.

Los resultados también coinciden, aunque en menor medida, con los hallazgos del estudio de Paulina-Osorio et al., (2015), donde se analiza la deforestación en una cuenca del estado de Guerrero, en el sur de México. Los autores establecieron que factores como la altitud, distancia a carreteras y distancia a localidades parecían tener mayor importancia en el proceso de pérdida de bosques, observando en este caso, un peso menor para factores estrictamente sociodemográficos. También coinciden parcialmente con el trabajo de López-Barrera, Manson, \& Landgrave (2014), desarrollado en el estado de Veracruz, por la costa atlántica de México. Estos autores encuentran mayor probabilidad de deforestación en áreas cercanas a polígonos de uso pecuario, pero en zonas con baja pendiente, y cercanas también a infraestructura hidráulica, que podría facilitar la irrigación agrícola.

En particular la relación existente entre tenencia de la tierra y degradación/deforestación, que obtuvo el valor de coeficiente más alto, contrasta con lo observado en otras regiones de México, donde son otros tipos de tenencia los que parecen asociarse más con la pérdida de cubierta vegetal. Los vínculos entre tenencia de la tierra y deforestación en este país han sido objeto de un fuerte debate, pues hay autores que mencionan a la propiedad ejidal como el tipo de tenencia más vinculado a la deforestación (Bonilla-Moheno et al., 2013) -lo que es contrario a nuestros hallazgos- mientras que otros indican que la tenencia ejidal sólo favorece la deforestación cuando las tierras ejidales son privatizadas (DiGiano, Ellis, \& Keys, 2013). Aún no hay un hallazgo concluyente, y faltan estudios más específicos sobre la relación de esta variable con la degradación y pérdida de cubierta vegetal (Skutsch et al., 2014).

Existen estudios que indican que las condiciones socioeconómicas, e incluso los rasgos socio-culturales de los habitantes pueden jugar un papel prominente en la conservación de los recursos naturales, pues subyacen a las reglas de manejo de recursos que son de propiedad común (Ceddia, Gunter, \& Corriveau-Bourque, 2015). En la zona de estudio por ejemplo, las áreas de propiedad ejidal se caracterizan por una importante proporción de población indígena, de la etnia Tarahumara, que posee una relación de ética con la naturaleza distinta a la observada entre la población mestiza, predominante en las áreas de propiedad privada en la región. En Latinoamérica se ha encontrado que la población indígena observa en general una lógica de no acumulación de riqueza, que puede llevarlos, si los factores institucionales lo favorecen, a realizar un manejo más sustentable de sus recursos forestales (Ceddia, Gunter, \& CorriveauBourque, 2015). Así, aunque la población Tarahumara de la región usualmente padece condiciones severas de pobreza y marginación (Messer, 2013), su presencia representa una fortaleza para la conservación de las masas forestales.

Por lo que se refiere al vínculo entre marginación y degradación-deforestación de cubiertas forestales, un enfoque reduccionista del problema llevaría a pensar que hay una relación inequívoca entre pobreza y degradación de los recursos naturales. Se trata de un supuesto que ya se ha debatido en la literatura académica (Daregot et al., 2015), pero hay opiniones divergentes respecto a la relación que existe entre dicha variable y la perdida de cobertura vegetal (Aggrey et al., 2010). Sin descartar que la pobreza ha sido una fuerza motriz del deterioro ambiental en otros escenarios geográficos (Ravnborg, 2003), y que podría serlo aquí, en la zona debe reconocerse que existen bandas delincuenciales que han obligado, bajo la 
Bravo Peña L. C., Torres Olave M. E., Alatorre Cejudo L. C., Castellanos Villegas A. E., Moreno Murrieta R.. L, Granados Olivas A., Uc Campos M., González León M., Wiebe Quintana L. C. (2017): "Áreas probables de degradación-deforestación de la cubierta vegetal en Chihuahua, México. Una exploración mediante regresión logística para el período 1985-2013", GeoFocus (Artículos), $n^{\circ}$ 20, p. 109-137. ISSN: 1578-5157 http://dx.doi.org/10.21138/GF.545

ley de plata o plomo, la deforestación de predios entre los propietarios ejidales o privados. En esta dinámica, los delincuentes aparentemente se aprovechan de la indefensión legal en la que se encuentran las localidades más marginadas, revelando con ello que los vínculos entre pobreza y degradación no son lineales ni directos. Dichos vínculos también se generan por la ausencia de autoridad y las debilidades socio-institucionales ligados a una situación de pobreza, que favorecen la impunidad de los delincuentes, y el atropello de los propietarios del bosque, por su carencia de medios materiales y legales para defenderse. Este factor podría tener mucho peso en la degradación-deforestación de la cubierta vegetal, pero debe explorarse con métodos complementarios a los empleados en este trabajo. Se requiere trabajo de campo desde una perspectiva sociológica o antropológica, para entender mejor el fenómeno.

Lo anterior también cobra vigencia en virtud del desfase observado entre los datos de marginación empleados en este trabajo, y las fechas correspondientes a la cartografía más antigua. En este ejercicio se utilizó la medición de marginación correspondiente al año de 1995, y en un sentido estricto, las condiciones de marginación en 1985 pudieron ser distintas a las condiciones una década después. Este desfase puede ser importante a la hora de interpretar los resultados, pero se ignora en que magnitud porque no hay datos para respaldarlo. Se sabe que la marginación juega un papel importante en los procesos de deforestación en México (MoralesBarquero, 2015 y Pineda Jaimes et al., 2009), pero establecer con claridad qué importancia tenía en la década de los 80’s en Chihuahua, requiere un acercamiento distinto al implementado en este trabajo. Precisa la revisión de estadísticas, censos de la época, etc., a fin de reconstruir en la medida de lo posible, la interacción entre esta variable y el proceso de deforestación. Dicho acercamiento deberá desarrollarse por especialistas en el área, con el propósito de esclarecer la relación precisa de esta variable y la degradación de cubiertas en la historia del estado. Por lo pronto, justifica la realización de nuevos ensayos de RLO, con datos socioeconómicos más actualizados, a efectos de modelar en forma más realista el papel que juegan dichos factores en la pérdida de cubierta vegetal.

Nuestros hallazgos se suman a los de otros trabajos que ponen en perspectiva la idoneidad de técnicas como la RLO para modelizar procesos como la deforestación, e identificar las causas asociadas con este proceso. Esta importancia se ha mencionado en distintos ejercicios de modelación de cambio de uso del suelo en México (Geoghegan et al., 2001; Mas, Puig, Palacio, \& Sosa-López, 2004; Vance \& Geoghegan, 2004); pero la diversidad de resultados fortalece la idea, de que es necesario modelizar para cada localidad o región particular, pues la importancia de un factor en una región, no necesariamente es equivalente o similar en otra (Angelsen \& Kaimowitz, 1999). Diferencias en el medio físico, o diferencias en las condiciones de carácter cultural, o de marginación o desarrollo humano, o incluso factores como subsidios gubernamentales y precio de productos agrícolas no considerados en este trabajo, pueden tener un papel muy relevante de una localidad a otra. Con todo, en este caso particular constituyen hallazgos que pueden orientar el trabajo de otras disciplinas, como la sociología, antropología, economía, necesarias para comprender el hilado fino que subyace a los vínculos entre factores socioeconómicos y la degradación o pérdida de las cubiertas forestales.

\section{Conclusiones.}

Los resultados de regresión obtenidos mostraron que es posible predecir con precisión la distribución de superficies susceptibles de degradación-deforestación en el área de estudio. El valor de P-seudo $\mathrm{R}^{2}$ resultante de regresar los procesos de degradación-deforestación contra las variables explicativas fue de 0.24 , que es muy adecuado de acuerdo a los parámetros del 
Bravo Peña L. C., Torres Olave M. E., Alatorre Cejudo L. C., Castellanos Villegas A. E., Moreno Murrieta R.. L, Granados Olivas A., Uc Campos M., González León M., Wiebe Quintana L. C. (2017): "Áreas probables de degradación-deforestación de la cubierta vegetal en Chihuahua, México. Una exploración mediante regresión logística para el período 1985-2013", GeoFocus (Artículos), $n^{\circ}$ 20, p. 109-137. ISSN: 1578-5157 http://dx.doi.org/10.21138/GF.545

método. Apunta en la misma dirección el valor de ROC alcanzado (0.85), pues este indica que el modelo predice la degradación-deforestación con una precisión muy aceptable, y superior a lo que se obtendría sólo al azar. Dicho resultado fortalece los hallazgos del trabajo, y corrobora la validez del modelo obtenido. Este sugiere que las probabilidades más elevadas de degradacióndeforestación se observan hacia el poniente de la zona de estudio, y en zonas muy localizadas del oriente de la misma. En estas zonas, las condiciones espaciales de las variables explicativas favorecen el proceso de degradación y pérdida de cubierta vegetal.

Todas las variables empleadas resultaron importantes para modelizar y predecir la probabilidad de degradación y deforestación de la cubierta vegetal, pero hay algunas que tienen un peso evidentemente mayor. Destacan por el valor y el signo de los coeficientes variables como tenencia de la tierra, marginación, cambio en la marginación durante el periodo 19952010, aptitud agrológica del suelo y pendiente. Dichos coeficientes y su signo revelan que la degradación y deforestación se incrementa en zonas donde predomina la propiedad privada, con alta marginación, con elevada pendiente y con un suelo relativamente adecuado para el desarrollo de actividades agrícolas. Como la marginación se relaciona con pobreza, esto sugiere que la degradación y pérdida de cubierta vegetal ocurre básicamente en zonas deprimidas económicamente, pero en áreas que presentan condiciones edáficas apropiadas para el desarrollo de cultivos agrícolas. Adicionalmente, como la degradación-deforestación se acentúa en áreas de alta pendiente, puede deducirse que los nuevos espacios agrícolas se abren en zonas marginales para el desarrollo de esta actividad, pues probablemente las áreas con condiciones fisiográficas más apropiadas, usualmente cercanas a localidades y con accesibilidad debido a la existencia de caminos, ya se utilizan con este fin desde hace muchos años.

Este modelo cuantifica la importancia de las causas asociadas a la perdida y degradación de cubierta vegetal, por lo que permite mejorar la regulación ambiental de las actividades que contribuyen con este proceso, mitigar las causas en áreas que no se han degradado o deforestado, y proponer soluciones en zonas que ya perdieron su cubierta vegetal. Su utilidad pone en perspectiva la idoneidad de técnicas como la RLO para modelizar procesos como la deforestación, e identificar las causas asociadas con este proceso. Facilitan la conservación ambiental de la cubierta vegetal, pero también permiten orientar el trabajo de otras disciplinas, como la sociología, la antropología y la economía, necesarias para comprender el hilado fino que subyace a los vínculos entre factores socioeconómicos y la degradación o pérdida de las cubiertas forestales.

Con todo, es importante señalar que el fenómeno de la degradación-deforestación en esta región de México es muy complejo. Nuevas exploraciones deberán realizarse, considerando factores adicionales no incluidos en esta primera exploración. El papel de los mercados agrícolas, los subsidios a la producción agropecuaria, el rol específico que juega el narcotráfico, el papel que puede desempeñar la cultura de los grupos involucrados, etc.; son aspectos que deben incluirse para entender $-\mathrm{y}$ eventualmente predecir- con más precisión la pérdida de cubierta forestal.

\section{Bibliografía.}

Aggrey, N., Wambugu, S., Karugia, J., \& Wanga, E. (2010): "An Investigation of the Poverty- Environmental Degradation Nexus: A Case Study of Katonga Basin in Uganda", Research Journal of Environmental and Earth Science, 2,2, 82-88. 
Bravo Peña L. C., Torres Olave M. E., Alatorre Cejudo L. C., Castellanos Villegas A. E., Moreno Murrieta R.. L, Granados Olivas A., Uc Campos M., González León M., Wiebe Quintana L. C. (2017): "Áreas probables de degradación-deforestación de la cubierta vegetal en Chihuahua, México. Una exploración mediante regresión logística para el período 1985-2013”, GeoFocus (Artículos), $n^{\circ}$ 20, p. 109-137. ISSN: 1578-5157 http://dx.doi.org/10.21138/GF.545

Aguilar, E. S. (2013): Conservación de la cubierta vegetal en el área natural protegida (ANP) Tutuaca. Evaluación con métodos de clasificación espectral. Tesis Licenciatura en Geoinformática, Ciudad Juárez, Universidad Autónoma de Ciudad Juárez.

Angelsen, A., \& Kaimowitz, D. (1999): "Rethinking the Causes of Deforestation: Lessons from Economic Models", The World Bank Research Observer, 14,1, 73-98.

Arekhi, M. (2013): "Modeling spatial pattern of deforestation using GIS and logistic regression: A case study of northern Ilam forests, Ilam province, Iran", African Journal of Biotechnology, 10,72, 16236-16249.

Bonilla-Moheno, M., Redo, D. J., Aide, T. M., Clark, M. L., \& Grau, H. R. (2013): "Vegetation change and land tenure in Mexico: A country-wide analysis", Land Use Policy, 30,1, 355-364.

Ceddia, M. G., Gunter, U., \& Corriveau-Bourque, A. (2015): "Land tenure and agricultural expansion in Latin America: The role of Indigenous Peoples and local communities forest rights", Global Environmental Change, 35, 316-322.

Clark, W.A., and P.L. Hosking, (1986): Statistical Methods for Geographers. New York, John Wiley \& Sons.

CONAGUA, 2015. Registro público de derechos del agua. México D.F. Subdirección de Información Geográfica del Agua, Comisión Nacional del Agua.

CONABIO, (2001): Grados de marginación a nivel localidad, 1995. México D.F. Comisión Nacional para el Conocimento y Uso de la Biodiversidad. Disponible en: http://www.conabio.gob.mx/informacion/gis/

CONABIO, (2012): Grados de marginación a nivel localidad, 2010. México D.F. Comisión Nacional para el Conocimento y Uso de la Biodiversidad. Disponible en: http://www.conabio.gob.mx/informacion/gis/

Basse, R. M., Omrani, H., Charif, O., Gerber, P., \& Bódis, K. (2014): "Land use changes modelling using advanced methods: Cellular automata and artificial neural networks. The spatial and explicit representation of land cover dynamics at the cross-border region scale", Applied Geography, 53, 160-171.

Daregot, B., Ayalneh, B., Belay, K., \& Degnet, A. (2015): "Poverty and Natural Resources Degradation: Analysis of their Interactions in Lake Tana Basin, Ethiopia", Journal of International Development, 27,4, 516-527.

DiGiano, M., Ellis, E., \& Keys, E. (2013): "Changing Landscapes for Forest Commons: Linking Land Tenure with Forest Cover Change Following Mexico's 1992 Agrarian CounterReforms", Human Ecology, 41,5, 707-723.

Eastman, J. R. (2012): Idrisi Selva Manual. Boston Ma, Clark University.

Eugenio, F. C., Rosa dos Santos, A., Fiedler, N. C., Ribeiro, G. A., da Silva, A. G., Juvanhol, R. S. Schettino V. R., Marcatti G. E., Domingues, G. F., Alves Dos Santos G. MAD, Pezzopane JEM, Pedra B. D, Banhos A., Martins, L. D. (2016): "GIS applied to location of fires detection towers in domain area of tropical forest", Science of The Total Environment, 562, 542-549.

Fagerholm, N., Oteros-Rozas, E., Raymond, C. M., Torralba, M., Moreno, G., \& Plieninger, T. (2016): "Assessing linkages between ecosystem services, land-use and well-being in an agroforestry landscape using public participation GIS", Applied Geography, 74, 30-46.

García, E. (1973): Modificaciones al sistema de clasificación climática de Köppen: para adaptarlo a las condiciones de la República Mexicana. México D.F. Instituto de Geografía, UNAM. 
Bravo Peña L. C., Torres Olave M. E., Alatorre Cejudo L. C., Castellanos Villegas A. E., Moreno Murrieta R.. L, Granados Olivas A., Uc Campos M., González León M., Wiebe Quintana L. C. (2017): "Áreas probables de degradación-deforestación de la cubierta vegetal en Chihuahua, México. Una exploración mediante regresión logística para el período 1985-2013”, GeoFocus (Artículos), $n^{\circ}$ 20, p. 109-137. ISSN: 1578-5157 http://dx.doi.org/10.21138/GF.545

Geoghegan, J., Villar, S. C., Klepeis, P., Mendoza, P. M., Ogneva-Himmelberger, Y., Chowdhury, R. R., Turner, B. L., Vance, C. (2001): "Modeling tropical deforestation in the southern Yucatán peninsular region: comparing survey and satellite data. Agriculture, Ecosystems \& Environment, 85,1-3, 25-46.

Hall, P. G. \& Hyndman, R. J. (2003): "An Improved Method for Bandwidth Selection When Estimating ROC Curves", Statistics and Probability Letters 64,2, 181-189.

Hall, P. G., Hyndman, R. J. \& Fan, Y. (2004): "Nonparametric Confidence Interval for Receiver Operating Characteristic Curve", Biometrika 91,3, 743-750.

Hillerkus, T. (1992): "Economía, Política y Orden Social de los Tarahumaras en la época prehispánica y colonial", Estudios de Historia Novohispana, 12, 9-62.

INEGI. (2001). Carta de uso de suelo y vegetación escala 1:250 000, serie I. Datos en formato vectorial generados por CETENAL para las ortofotos e imágenes de 1985. Aguascalientes, Aguascalientes.

INEGI. (2007). Carta Edafológica H13-10. ESCALA 1:250,000. Instituto Nacional de Estadística, Geografía e Informática.

INEGI. (2013a). Continuo de Elevaciones Mexicano 3.0. Aguas Calientes, México. Instituto Nacional de Estadística, Geografía e Informática. Disponible en: http://www.inegi.org.mx/geo/contenidos/datosrelieve/continental/descarga.aspx

INEGI. (2013b). Conjunto de datos vectoriales de uso de suelo y vegetación escala 1:250 000, serie V (capa unión)', escala: 1:250000. edición: 2a., Aguas Calientes, México. Instituto Nacional de Estadística, Geografía e Informática. Disponible en: http://www.inegi.org.mx/geo/contenidos/recnat/usosuelo/Default.aspx

INEGI. (2015): Guía para la interpretación de cartografía de Uso del suelo y vegetación. Escala 1:250000, Serie V. Aguas Calientes, México. INEGI..

Klepeis, P., \& Vance, C. (2003): "Neoliberal Policy and Deforestation in Southeastern Mexico: An Assessment of the PROCAMPO Program", Economic Geography, 79(3), 221-240.

Kumar, R., Nandy, S., Agarwal, R., \& Kushwaha, S. P. S. (2014): "Forest cover dynamics analysis and prediction modeling using logistic regression model", Ecological Indicators, 45, 444-455.

Lebgue, K. T., Quintana, M.G., y R. Soto, C. (2015): Pinos y Encinos de Chihuahua. Chihuahua, México. Universidad Autónoma de Chihuahua.

Liénard, J. F., Gravel, D., \& Strigul, N. S. (2015): "Data-intensive modeling of forest dynamics", Environmental Modelling \& Software, 67, 138-148.

Lin, C., Thomson, G., Hung, S.-H., \& Lin, Y.-D. (2012): "A GIS-based protocol for the simulation and evaluation of realistic 3-D thinning scenarios in recreational forest management", Journal of Environmental Management, 113, 440-446.

Marín, S. L., Nahuelhual, L., Echeverría, C., \& Grant, W. E. (2013): "Projecting landscape changes in southern Chile: Simulation of human and natural processes driving land transformation", Ecological Modelling, 222,15, 2841-2855.

Mas, J. F., Puig, H., Palacio, J. L., \& Sosa-López, A. (2004): "Modelling deforestation using GIS and artificial neural networks". Environmental Modelling \& Software, 19,5, 461471.

Messer, S. (2013): "Tackling Maternal Mortality in the Sierra Tarahumara, Mexico: A Community Based Intervention”, Journal of Obstetric, Gynecologic \& Neonatal Nursing, 40, Supplement 1, S10. 
Bravo Peña L. C., Torres Olave M. E., Alatorre Cejudo L. C., Castellanos Villegas A. E., Moreno Murrieta R.. L, Granados Olivas A., Uc Campos M., González León M., Wiebe Quintana L. C. (2017): "Áreas probables de degradación-deforestación de la cubierta vegetal en Chihuahua, México. Una exploración mediante regresión logística para el período 1985-2013”, GeoFocus (Artículos), $n^{\circ}$ 20, p. 109-137. ISSN: 1578-5157 http://dx.doi.org/10.21138/GF.545

Mertens, B., Kaimowitz, D., Puntodewo, A., Vanclay, J. K., \& Mendez, P. (2004): "Modeling deforestation at distinct geographic scales and time periods in Santa Cruz, Bolivia" International Regional Science Review, 27(3).

Morales-Barquero, L., Borrego, A., Skutsch, M., Kleinn, C., \& Healey, J. R. (2015): "Identification and quantification of drivers of forest degradation in tropical dry forests: A case study in Western Mexico", Land Use Policy, 49, 296-309.

$\mathrm{Mu}, \mathrm{L}$. (2009): Thiessen Polygon A2 - Kitchin, Rob. En N. Thrift (Ed.), International Encyclopedia of Human Geography (pp. 231-236). Oxford. Elsevier.

Pardo M., A., y Ruíz D., M. (2002): SPSS 11: Guía para el análisis de datos. (Segunda Ed.). México D.F.: McGraw-Hill.

Paulina Osorio, L., Mas, J.-F., Guerra, F., \& Maass, M. (2015): “Análisis y modelación de los procesos de deforestación: un caso de estudio en la cuenca del río Coyuquilla, Guerrero, México”, Investigaciones Geográficas, Boletín del Instituto de Geografía, 2015,88, 60-74.

Pérez, J. L., Villalobos, M. A., Rosete, F., Navarro, E., Salinas, E. y Remond, R. (2013): Procesos del cambio de la vegetación y del uso del suelo, 1976-2008. México, D. F, INESEMARNAT.

Pérez-Vega, A., Mas, J.-F., \& Ligmann-Zielinska, A. (2012): "Comparing two approaches to land use/cover change modeling and their implications for the assessment of biodiversity loss in a deciduous tropical forest", Environmental Modelling \& Software, 29,1, 11-23.

Pineda Jaimes, N. B., Bosque Sendra, J., Gómez Delgado, M., \& Plata Rocha, W. (2009): "Análisis de cambio del uso del suelo en el Estado de México mediante sistemas de información geográfica y técnicas de regresión multivariantes: Una aproximación a los procesos de deforestación", Investigaciones geográficas, 69, 33-52.

Pir, B. M. (2015): "Deforestation modelling using logistic regression and GIS", Journal of Forest Science, 61,5, 193-199.

Porras, E. (1999): "La Sierra Tarahumara de Chihuahua (México): una región multiétnica y pluricultural", Scripta Nova Revista Electrónica de Geografía y Ciencias Sociales., 45,51. Recuperado a partir de http://www.ub.edu/geocrit/sn-45-51.htm

Pontius, R. G., Shusas, E., \& McEachern, M. (2004): "Detecting important categorical land changes while accounting for persistence", Agriculture, Ecosystems \& Environment, 101, 251-268.

Priego, A., Bocco, G., Mendoza, M., \& Garrido, A. (2008): Propuesta para la generación semiautomatizada de unidades de paisajes. Fundamentos y Métodos. Mexico D.F, SEMARNAT-INE.

RAN. (2012): Mapa Digital de Tenencia de la Tierra en Chihuahua, Cd. Chihuahua, Registro Agrario Nacional.

Ravnborg, H. (2003): "Poverty and environmental degradation in the Nicaraguan Hillsides", World Development, 31,11, 1933-1946.

Rogerson, P. A. (2010). Statistical Methods for Geography: A Student's Guide (Third Edition), London, SAGE Publications Ltd.

Roy, C. (2006): "Landscape change in the Calakmul Biosphere Reserve, Mexico: Modeling the driving forces of smallholder deforestation in land parcels", Applied Geography, $26,2,129-152$.

Rzedowski, J. (1978): Vegetación de México. México D.F., Editorial Limusa.

Semeraro, T., Mastroleo, G., Aretano, R., Facchinetti, G., Zurlini, G., \& Petrosillo, I. (2016): "GIS Fuzzy Expert System for the assessment of ecosystems vulnerability to fire in 
Bravo Peña L. C., Torres Olave M. E., Alatorre Cejudo L. C., Castellanos Villegas A. E., Moreno Murrieta R.. L, Granados Olivas A., Uc Campos M., González León M., Wiebe Quintana L. C. (2017): "Áreas probables de degradación-deforestación de la cubierta vegetal en Chihuahua, México. Una exploración mediante regresión logística para el período 1985-2013”, GeoFocus (Artículos), $n^{\circ}$ 20, p. 109-137. ISSN: 1578-5157 http://dx.doi.org/10.21138/GF.545

managing Mediterranean natural protected areas", Journal of Environmental Management, 168, 94-103.

Serra, P., Pons, X., \& Saurí, D. (2008): "Land-cover and land-use change in a Mediterranean landscape: A spatial analysis of driving forces integrating biophysical and human factors", Applied Geography, 28(3), 189-209.

Shalaby, A., \& Tateishi, R. (2007): "Remote sensing and GIS for mapping and monitoring land cover and land-use changes in the Northwestern coastal zone of Egypt", Applied Geography, 27,1, 28-41.

Skutsch, M., Mas, J. F., Bocco, G., Bee, B., Cuevas, G., \& Gao, Y. (2014): "Deforestation and land tenure in Mexico: A response to Bonilla-Moheno et al", Land Use Policy, 39, 390-396.

Strahler, A. H., \& Strahler, A. (2004): Physical Geography: Science and Systems of the Human Environment (3 edition). New York, Wiley \& Sons.

Vance, C., \& Geoghegan, J. (2004): "Modeling the Determinants of Semi-Subsistent and Commercial Land Uses in an Agricultural Frontier of Southern Mexico: A Switching Regression Approach" International Regional Science Review, 27(3), 326-347.

Velázquez, A., Mas J.F., Díaz J.R., Mayorga R., Alcántara P.C., Castro R., Fernández T., Bocco G., Ezcurra E. y J.L. Palacio. (2002): "Patrones y tasas de cambio de uso del suelo en México", Gaceta Ecológica, 62, 21-37.

Vida Nueva. (2015). "Ecocidio en la Tarahumara, crimen organizado impune" Recuperado el 11 de octubre de 2016, a partir de http://www.revistavidanueva.mx/content/ecocidio-en-la-tarahumara-crimen-organizado-impune

Wilson, K., Newton, A., Echeverría, C., Weston, C., \& Burgman, M. (2005): "A vulnerability analysis of the temperate forests of south central Chile", Biological Conservation, $122,1,9-21$.

Zambelli, P., Lora, C., Spinelli, R., Tattoni, C., Vitti, A., Zatelli, P., \& Ciolli, M. (2012): "A GIS decision support system for regional forest management to assess biomass availability for renewable energy production", Environmental Modelling \& Software, 38, $203-213$.

Zheng, X., \& Fan, R. (2015): "Predicting Probability of Deforestation in Belize". Atlas of Maine, 2015,2.

Zócalo. (2016). "Acusan incendio intencional en bosque de la Sierra Tarahumara [Chihuahua]" | Periódico Zócalo. -07/05/2016. Recuperado el 11 de octubre de 2016, a partir de http://www.zocalo.com.mx/seccion/articulo/acusan-incendio-intencional-en-bosque-de-la-sierratarahumara-1462648055 
